\title{
(Un)doing gender in den räumlichen Arrangements der Kita
}

\author{
Franziska Vogt
}

Um Alltagspraktiken des doing und undoing gender in der Kita zu beschreiben, muss auch der Raum einbezogen werden. Wie die Kita als gendered organisation (Acker 1990) verstanden wird, zeigt sich auch der Kitaraum als gendered. Die Pädagogik der frühen Kindheit weist dem Raum und den Spielmaterialien eine hohe Bedeutung zu. Es ist deshalb in der Erforschung von (un)doing gender in der Kita notwendig, dass der Raum, als von den Kinderbetreuenden eingerichteter Raum mit Aktivitäten- und Spielecken und dem angebotenen Spielmaterial, ebenfalls analysiert wird.

\subsection{Gender im Raum}

Räume sind in der Erforschung von doing und undoing gender von Interesse, da sie „Konstruktionsmittel sozialer Wirklichkeit“ sind (Hinz 2012, S. 51), die Aspekte des institutionalisierten Arrangements zeigen (Goffman 1994). Sie können als Genderismen, als institutionell zur Verfügung stehende Infrastrukturen für das situativ mögliche doing und undoing gender im Sinn von Dramatisierungen und Entdramatisierungen verstanden werden. Genderismen beziehen sich auf an ein Geschlecht gebundene Erwartungen und Verhaltensweisen, mit denen auch die Zugehörigkeit zu einem Geschlecht inszeniert werden kann. Stereotypes Wissen über Geschlechterdifferenzen wird inszeniert und dadurch erneut festgeschrieben. Frehse (2016) verdeutlicht, dass im Raumbegriff von Goffman nicht der Raum als physisches Setting, sondern die durch den Raum ermöglichten

\section{F. Vogt $(\bowtie)$}

Pädagogische Hochschule St. Gallen, St. Gallen, Schweiz

E-Mail: franziska.vogt@phsg.ch 
sozialen Interaktionen im Vordergrund stehen. Der Raum ist nicht nur physische Umgebung, sondern ,physisches Konditionierungsmedium, Zeichen und Idiom von Interaktionen“ (Frehse 2016, S. 11).

Raum wird als sozialer Raum verstanden, der sozial konstruiert und durch Performanz kreiert ist und der von Neuem zukünftige Handlungen ermöglicht; dabei werden gewisse Handlungen suggeriert und andere verhindert (Lefèbvre 1991). Räume sind mehr als Behälter, sie sind sozial konstruiert, sie zeigen soziale Beziehungen und beeinflussen Menschen und Beziehungen (Löw 2008). In der Raumsoziologie lassen sich zwei Orientierungen unterscheiden: Raum als Behälterraum oder Raum als Beziehungsraum. Eine Raumanalyse sollte darum sowohl die Strukturierungsmacht des Raums wie auch seine Prozesshaftigkeit berücksichtigen (Löw und Sturm 2019). Der Raum als sozialer Raum repräsentiert soziale Beziehungen und soziale Ordnungen und hat weiter eine Orientierungsfunktion für das Soziale (Kasüschke 2016). Damit wird Raum als räumliche Praxis und performativ betrachtet, eine Perspektive, die für eine Analyse hinsichtlich doing und undoing gender eine Basis bildet (Beebe et al. 2017). Wassermann und Frenkel (2015) unterscheiden im Anschluss an Lefèbvre (1991) für ihre Analyse vergeschlechtlichter räumlicher Praktiken den geplanten Raum von der räumlichen Praxis sowie dem gelebten Raum. Während der geplante Raum die Diskurse und die Raumrepräsentationen zum Beispiel der Architektinnen und Architekten oder auch der an der Planung beteiligten Fachpersonen beschreibt, geht es bei der räumlichen Praxis um die konkrete Umsetzung mittels materieller Artefakte und routinierter Praktiken sowie im gelebten Raum um die Interpretationen derer, die den Raum schließlich nutzen.

Aus Geschlechterperspektive sind Bildungsräume bisher insbesondere hinsichtlich der Raumnutzung von Mädchen und Jungen untersucht worden. So zeigen Breidenstein und Kelle (1998) wie auch Thorne (1992/2007), dass die Sitzordnung in Schulen einer Einteilung nach gender folgt. Wenn Kinder selbst wählen, setzen sich Mädchen neben Mädchen, Jungen neben Jungen. Thorne (1992/2007) beobachtete eine hälftige Aufteilung des Unterrichtsraums. Auch Räume wie öffentliche Spielplätze, die teilweise ohne Aufsicht von Erwachsenen genutzt werden, zeigen Raumaufteilungen entlang der Geschlechterkategorie: Karsten (2003) analysierte mittels strukturierter ethnografischer Beobachtung, wo sich Kinder aufhielten. Es zeigte sich, dass die Spiele der Jungen, fokussiert auf Fußball, sehr viel mehr Raum einnahmen als die vielfältigen Spiele der Mädchen. Weiter wurde beobachtet, wie durch die räumliche Aufteilung doing gender verstärkt und undoing gender erschwert wird. Als Beispiel dafür wird ein Mädchen erwähnt, das gern Fußball spielt, aber nicht mit den Jungen mitspielen kann. Die Analyse zeigt gender divisions (geschlechtsspezifische Aufteilungen) in der 
Raumnutzung, aber auch in der zeitlichen Nutzung: Jungen kontrollierten ein gröBeres Territorium auf dem Spielplatz und hielten sich am Abend auch viel länger auf den Plätzen auf (Karsten 2003).

Mit einem Blick auf die Raumpraktiken fokussiert die Analyse von (un)doing gender auf organisationale Aspekte: Jede Organisation ist geprägt von Geschlechtervorstellungen, Organisationen können als gendered organizations (vergeschlechtliche Organisationen) analysiert werden (Acker 1990), die sich wiederum in den Raumpraktiken zeigen. Rohrmann (2009, S. 34) bezeichnet Kindergärten „,als Gärten der Frauen“, und verweist auf die „,impliziten ,Botschaften“ von Räumen und Materialien“. Dieken und Rohrmann (2003) analysieren die Farbwahl, die Art der Dekorationen, die zahlreichen Tücher und Kuschelecken sowie das Bastelangebot als eine weiblich geprägte Ästhetik. Ebenfalls zugespitzt drückt dies Rabe-Kleberg (2003, S. 64) aus, indem sie Kindergärten mit Verweis auf die Raumgestaltung als ,weibliche Arenen“ beschreibt.

Doing gender und undoing gender in der Kita erfolgt in Räumen, die von den Kinderbetreuenden für die Kinder vorbereitet und gestaltet werden. Dem Raum kommt in der Pädagogik, und besonders in der frühen Bildung eine wichtige Rolle zu. Für die Untersuchung von (un)doing gender in den Raumpraktiken der Kita ist es darum wesentlich, auch die dort präsenten Spielmaterialien einzubeziehen. Im ersten Teil des Kapitels wird zunächst die Rolle des Raums für die Pädagogik dargelegt, insbesondere für die Pädagogik der frühen Kindheit. Weiter wird die Bedeutung des Spielmaterials für die frühe Bildung sowie für die im Spielmaterial deutlich werdenden Genderismen fokussiert, dies auf der Grundlage der Forschung zu Geschlechterstereotypen (Eckes 2008).

\subsection{Bildungsräume}

Räume sind Räume mit einer Geschichte; in ihnen sind nicht nur die Ordnung der Interaktion nach Goffman (1994), sondern auch das Werden der Räume in der Zeit sichtbar. Wenn Praktiken und Alltagskulturen in Bildungsinstitutionen untersucht werden sollen, zeigt sich deutlich, dass sich diese in Anlehnung an Lefèbvre (1991, S. 84) in „Abhängigkeit von Zeit und Raum“ beschreiben lassen. Sie sind zugleich eine normative, wie auch eine interpretative Realität (Jäger 2008). Räume sind mit normativen Erwartungen an das soziale Verhalten der Personen verknüpft - deutlich wird besonders bei Schulräumen und Hochschulauditorien, wie sehr die Räume die normative Erwartung dessen, was Schule und Universität verlangt, verkörpern (Blommaert 2013). Der Raum bestimmt, welches Verhalten erwartet und 
unterstützt wird und welches nicht und kann gar als Teil des ,heimlichen Lehrplans" bezeichnet werden (Dahlinger 2009, S. 247). Wie in der Soziologie wird auch in der Bildungswissenschaft von einem spatial turn gesprochen (Gulson and Symes 2007).

Der Gestaltung des Raums als Gestaltung des Lernangebots wird in der Kitaund Kindergartenpädagogik traditionell eine hohe Bedeutung zugemessen. Die Alltagspraxis in den Kitas soll darum als „,verräumlichend“ und als „,verräumlicht" analysiert werden (Kuhn 2013, S. 170). Wiederholt wird beispielsweise postuliert, dass es für effektives Lernen in Kita und Kindergarten auf die Umgebung ankomme (Crowther 2007). Andere halten dieser Betonung entgegen, dass der Raum zwar eine Basis für gelingende Bildungsprozesse sein kann, der Raum selbst dafür jedoch kein Garant ist; nötig dafür wäre die Verbindung von Raum und Didaktik (Hinz 2012).

Die intensive Auseinandersetzung mit Raum und Spielmaterial für die frühe Bildung wird seit Beginn des 19. Jahrhunderts geführt. Wolke beschrieb 1805 die Einrichtung von ,Spielzimmern“ in Bewahranstalten für Kinder bis drei Jahre und die Einrichtung von „Denklehrzimmern“ für die ,Vorbereitschule“, in denen spielend und aus eigenem Antrieb gelernt werden kann (Reyer und Franke-Meyer 2016). Fröbel definierte „Spielgaben“, um durch die Beeinflussung der Wahl des Spielmaterials Bildung zu ermöglichen (Erning 2004). Pestalozzi betonte die Bedeutung der Bildungsräume als Lebensräume der Kinder. Für die frühe Kindheit forderte Pestalozzi, dass frühe Bildung gänzlich in ,die vier Wände der Wohnstube des reinen häuslichen Lebens“ und in die „Hand der Mutter“ gelegt wird (Pestalozzi 1997, zit. in Stieve 2013, S. 94). Im pädagogischen Diskurs des 19. Jahrhunderts wurde der privat-familiale Haushalt als geeigneter Bildungsraum für die frühe Kindheit gesehen, die damals entstehenden Kinderkrippen, sogenannte Kinderbewahranstalten, als die aus einer Betreuungsnot entstandene weniger gute Lösung. So war der Grundgedanke einer der Pionierinnen der frühkindlichen Bildung, Henriette Schrader-Breymann, die ,Anstaltsatmosphäre der damaligen Einrichtungen durch möglichst große Familienähnlichkeit aufzulösen“ (Reyer und Franke-Meyer 2016, S. 171). In der Montessori-Pädagogik, die die frühe Bildung seit Beginn des 20. Jahrhunderts beeinflusst, ist die Raumgestaltung mit qualitativ hochwertigem und größenangepasstem Mobiliar wichtig, damit diese dem Kind Freiraum für die Entwicklung geben kann. Aufgabe der Fachpersonen ist es, die Lernumgebung für die Selbsttätigkeit des Kindes vorzubereiten (Pütz und Klein-Landeck 2019).

In der Pädagogik von Reggio Emilia gegen Ende des 20. Jahrhunderts wird dem Raum der Status eines „,dritten Pädagogen“ zugeschrieben. Der Raum wird dadurch zu einer pädagogischen Instanz, die die Interaktionen zwischen Kind, 
Lerngegenstand und Fachperson mitprägt (Dahlinger 2009). Dahlinger (2009, S. 248) postuliert, dass Räume bildend auf Kinder wirken, ob intendiert oder nicht. Auf Schäfer (2005, zit. in Franz und Vollmert 2005) wird verwiesen mit der Aussage, dass der Raum der erste Erzieher sei, da Räume immer da sind und Erfahrungen ermöglichen, auch wenn keine Fachpersonen präsent sind. Raum meint auch Außenräume wie Gärten oder auch der Wald. Reyer und Franke-Meyer (2016) interpretieren die Bewegung der Waldkindergärten und Waldkitas als eine weitere pädagogische Richtung, die dem Wald die Rolle eines Hauptakteurs im Bildungsprozess zuschreiben, um dadurch dem negativ besetzten, anstaltsförmigen Raum zu entgehen. Pädagogische Ansätze definieren sich demnach gerade in der frühen Bildung durch das Vorhandensein bestimmter Elemente der Raumgestaltung und Einrichtungsgegenstände, so beispielsweise die Pastelltöne in der Waldorfpädagogik, die Bewegungselemente wie Podeste und schiefe Ebenen für die Pädagogik nach Pikler oder die Spiegel für die Pädagogik der Reggio Emilia (Hoffmann 2013; Knauf 2019).

In der Auseinandersetzung mit den Bildungsräumen der frühen Kindheit wird auf die besonderen Bedürfnisse von Kleinkindern verwiesen. Für die Raumgestaltung wird empfohlen, die Perspektive des Kindes einzunehmen und die „Maßstäblichkeit des Kindes“ zu berücksichtigen (Franz und Vollmert 2005, S. 18). So seien die Kitaräume häufig zugleich zu groß und zu klein, denn die Wahrnehmung der jungen Kinder sei überfordert mit der Größe eines normalen Kitaraums, zugleich ist der Raum zu klein, wenn sich die übliche Anzahl Kinder in ihm bewegen (Mahlke 2003). Es brauche definierte, kleinere Nischen und Ecken für einzelne Kinder und Kindergruppen. Wesentliche Anregungen für die Raumgestaltung in Kitas könnten auch aus der Inklusionspädagogik kommen, die die Raumgestaltung für Kinder mit Seh- und Hörbeeinträchtigungen untersucht. Dabei wird stärker die Raumwahrnehmung und Wahrnehmungsentwicklung der Kinder in der Raumgestaltung berücksichtigt, eine Perspektive, die für die pädagogische Raumgestaltung insgesamt nützlich wäre (Gutknecht 2016). Für kleine Kinder bis drei Jahre ist die Raumgestaltung vom Boden und vom Bewegungsbedürfnis aus zu denken. Zudem muss die weitere Entwicklung mit einbezogen werden, das Kind sollte deshalb ,immer etwas mehr Raum zur Verfügung haben, als es ihn nutzen kann“ (von der Beek 2014, S. 66).

Kinder im Kitaalter haben schon eine sehr gute Repräsentation des Raums. Fünfjährige Kinder erinnerten sich in einem Experiment daran, welche Gegenstände nahe beieinander oder weiter voneinander entfernt sind. Dies gelingt ihnen dann besser, wenn sie den Raum in Eigenbewegung erfahren, sich also körperlich im Raum bewegen (Wilkening 2004). In einer Untersuchung in zehn Kitas 
in Deutschland mithilfe einer Raumqualitätsskala wurde zwischen einschränkenden und ausdrucksstarken Umgebungen unterschieden; als einschränkend werden Räume bezeichnet, in denen das Material in Schränken verstaut ist und kaum Spuren der Kinder (Zeichnungen, Fotos usw.) sichtbar sind; als ausdrucksstark gelten Räume mit sichtbaren und zugänglichen Materialien und einer Struktur von Funktionsecken und Lernbereichen (Knauf 2019). Für das Primarschulalter kann der Einfluss der Raumqualität auf den Lernerfolg quantifiziert werden. Mithilfe von Mehrebenenanalysen wurde festgestellt, dass die Raumqualität des Klassenzimmers, beurteilt anhand von Faktoren wie Licht, Flexibilität und weiteren, einen Einfluss auf den Lernfortschritt in Mathematik und Sprache von Primarschulkindern hat und bis etwa $10 \%$ der Varianz erklärt (Barrett et al. 2017).

In der Kita wird die klassische Raumgestaltung so beschrieben: Es hat eine Puppen- und Küchenecke, einen Maltisch, einen Bauteppich, und Tische für das zweite Frühstück und das Mittagessen (Kogel 2007, S. 12). Wie Kasüschke (2016) aufzeigt, werden auch in aktuellen Ansätzen der Kindergartenpädagogik Funktionsecken so eingerichtet, dass sie Bildungsgegenstände repräsentieren. In einer kindzentrierten, von der Selbstbildung des Kindes ausgehenden Kindergartenpädagogik stehen diese Funktionsecken bereit, damit das Kind von sich aus im Freispiel eine der Ecken für sein Spiel wählt (Kasüschke 2016).

In den letzten Jahren wurden in Deutschland, seit Neuerem auch in der Schweiz, offene Raumkonzepte ausprobiert. Das sogenannte offene System bedeutet für Kitas, dass sie gruppenübergreifend arbeiten und die vorhandenen Räume dafür nutzen, vielfältigere Bildungsangebote zur Verfügung zu stellen. Wie Kogel (2007) pointiert herausarbeitet, stellt sich die Frage, ob in jedem Gruppenraum eine Puppen- und Küchenecke, eine Bauecke und Tische für Spiele und für Essen eingerichtet sein soll, mehrmals in der ganzen Kita das gleiche, oder ob Räumen Tätigkeiten und Bildungsbereiche zugewiesen werden sollen, die dann von allen genutzt werden können. Dies soll nicht nur für den Bewegungsraum, sondern auch für Räume für Kreativität und für Forschen und Experimentieren erfolgen. Kogel (2007, S. 13) sieht darin eine „optimale Ausnutzung bestehender Ressourcen“. Sie betont, dass Kitas, die sich räumlichen Öffnungen zuwenden, dies als Weiterentwicklung positiv erleben. Auch im offenen System wird weiterhin die Rolle des Raums als Hauptakteur der frühkindlichen Bildung betont (Kasüschke 2016). Das offene System führt die Funktionsecken fort und anstelle der Ecken im Gruppenraum werden in den verschiedenen Funktionsräumen Bildungsbereiche repräsentiert. Das offene System löst die feste Gruppenstruktur zumindest teilweise auf, damit wird auch die Nachbildung der Familie in der Raumstruktur der Kita verringert. Das traditionelle System bildet in jedem 
Gruppenraum eine häusliche Umgebung ab, in einer Kita mit mehreren Gruppenräumen sind also wie in einem Mehrfamilienhaus mehrere Familienwohnungen mit ihren dazugehörigen Küchen und Sofas strukturell definiert. Im offenen System ist eher ein moderner Arbeitsort in einer großen Organisation ohne feste Arbeitsplätze oder ein Einkaufszentrum mit Läden, die die Kundinnen und Kunden zu sich in den Raum locken, die entsprechende Analogie. Kasüschke (2016, S. 191) beschreibt den Wechsel vom Gruppenraum mit Funktionsecken zu Funktionsräumen im offenen System als ein Wechsel des gesellschaftlichen Abbilds, von der ,heilen Familie“ zur postmodernen Gesellschaft, in der Kinder individuell von einer spezialisierten Bildungsinsel zur anderen wandern. Die Intensität und Kontroverse der Diskussion in der Praxis zeigt einmal mehr die hohe Bedeutung, die der Raumgestaltung in der Kita zugewiesen wird. Es mangelt jedoch an wissenschaftlich fundierten Untersuchungen zur Rolle des Raums in der Pädagogik der frühen Kindheit. Funktionsecken und ihre räumliche Zuordnung, wie auch die Funktionsräume und ihre Ausgestaltung durch die Kinderbetreuenden wurden als Arrangements, als geplanter Raum, noch kaum analysiert. Es stellt sich die Frage, wie die räumlichen Arrangements im geplanten Raum der Kita mit den Funktionsecken oder mit Funktionsräumen als institutionalisierte Arrangements Genderismen fortschreiben.

\subsection{Puppenstuben und Bauecken? Spiel, Spielmaterialien und gender}

Die Bedeutung des Raums, wie sie in den verschiedenen dargestellten pädagogischen Konzeptionen der frühen Bildung betont wird, geht einher mit einer Betonung des Spiels. Wenn das Spiel einbezogen wird, kommt der gelebte Raum, mit einer Analyse der bereitgestellten Spielangebote der Repräsentationsraum sowie die räumliche Praxis in den Blick (Lefèbvre 1991). Hauser (2005) nennt das Spiel den „Lernmodus der frühen Kindheit“. Mit Rückgriff auf verschiedene Forschungen zum kindlichen Spiel werden die folgenden Spielarten unterschieden: Funktionsspiel, Bewegungsspiel, Objekt- und Konstruktionsspiel, Regelspiel sowie Fantasie- und Rollenspiel (Hauser 2016). Für das Rollenspiel sind Requisiten wichtig, hierzu gehört das Hantieren mit Gegenständen (Andresen 2011).

Ein traditioneller und prominenter Rollenspielbereich ist die Puppenecke, die auch als Babyecke, Küchenecke oder Familienecke bezeichnet wird. Mit dem Einrichten einer Puppenecke wird die häusliche Wohnung, die an sich als erste Umgebung der Kinder einen starken, habituellen Einfluss hat, in der Kita erneut 
repräsentiert, sodass zum So-tun-als-ob-Spiel in Nachahmung der Erwachsenen aufgefordert wird (Stieve 2013). Nach Stieve (2013) prägte die Pädagogik von Henriette Schrader-Breymann, die ab 1873 einen Pestalozzi-Fröbel-Kinderhaus aufbaute (Berger 2000), die Spielorte in Kindergärten: sie richtete eine Küchenecke und eine Bauecke ein, um das häusliche Leben mit Küche und Werkstatt zu repräsentieren.

Auch in der aktuellen Fachliteratur für die Einrichtung von Kitas werden diese Konzepte übernommen und betont. So wird beispielsweise für das Rollenspiel vorgesehen, dass es möglichst realistisch Routinen aufbaut, die geübt werden können, so beispielweise ,Anziehen, Geschirrspülen, Baden von Babys... Die Kinder sollen lernen, wie man diese Dinge richtig ausführt" (Crowther 2007, S. 288). Die Materialien für das Rollenspiel werden um die Themen Küche, Babybad, Schlafbereich, Requisiten und Verkleidung aufgeführt (Crowther 2007). Die Auflistung umfasst in der überwältigenden Mehrheit weiblich konnotierte Tätigkeiten in Haushalt und Kinderbetreuung. Es fällt auf, dass auf dieser Liste nicht nur „Kleider, Röcke, Blusen“, sondern auch „Anzüge, Krawatten, Hemden“ (Crowther 2007, S. 299) gefordert werden. Auch van Dieken und Rohrmann (2003, S. 31) fragen, ob es ,Anzüge, Hüte, Krawatten und lange Mäntel, Uniformen, Bauarbeiterhelme, Laptops, Pfeil und Bogen, Kochmützen, Detektivausrüstungen und Jungenzeitschriften" in der Rollenspielecke hat. Und sie konstatieren: „An der Gestaltung und Ausstattung von Räumen wird immer eine kultur- und geschlechtsspezifische Auswahl von Materialen und Gegenständen sichtbar" (van Dieken und Rohrmann 2003, S. 26). Es ist davon auszugehen, dass die vorhandenen Requisiten auch für männliche Rollen im Rollenspiel eine Bedeutung haben (Franz und Vollmert 2005).

Für die Puppenecke sind Puppen zentral, zugleich ist das Verhältnis zwischen Mädchen, Jungen und Puppen ambivalent. Puppen und die Erzählungen über Mädchen und Puppen dienten der Vorbereitung auf die klassische weibliche Rolle als ,tüchtige Hausfrau, brave und treue Ehefrau und hingebungsvolle Mutter“ (Fooken 2019, S. 98). Das konkrete Puppenspiel enthält jedoch ,immer auch Widerständiges und Subversives“ (Fooken 2019, S. 98). Puppen seien Projektionsflächen, Alter-Ego und Begleiterin. So beobachtete auch Stieve (2013), dass aus verschiedenen Gegenständen im Spiel anderes wurde, so wurde ein Korken zu einem Schnitzel, die Puppen wurden achtlos auf den Herd gelegt, das Spiel wurde unterbrochen und die Spielthemen wechselten. Seine Schlussfolgerung: „Es bleibt eine Unbestimmtheit, weil die Ordnungen der Dinge und damit auch die sozialen Ordnungen im Handeln und Interagieren an ihren Grenzen erworben werden, in einer ständigen Grenzverschiebung des Möglichen mit allen dazugehörigen Konflikten“" (Stieve 2013, S. 103). 
Während diese Beobachtungen des kindlichen Spiels im „gelebten Raum“ (Lefèbvre 1991) als fluide und häufig auch subversiv beschrieben werden können, erscheinen die durch den Raum bereitgestellten Arrangements stark an traditionellen Rollenvorstellungen der Geschlechter orientiert zu sein. Es werden weiblich und männlich konnotierte Spielbereiche installiert:

„In nicht wenigen Kitas wird nach wie vor eine geschlechtsspezifische Raumaufteilung realisiert. So finden Jungen und Mädchen jeweils Raumbereiche vor, die ihnen entsprechend bestehender Rollenerwartungen zugedacht sind, wenngleich sie auch dem anderen Geschlecht grundsätzlich zugänglich sind. Jungen etwa finden eine für sie gedachte Bauecke, Mädchen eine zum Rollenspiel einladende Nachbildung häuslicher Realitäten, Jungen eine Bewegungsecke, Mädchen einen Kreativbereich. (Braun und Steffes 2013, S. 15)“

Nicht nur in der Kita besteht diese Geschlechtersegregation, sondern möglicherweise noch stärker in den kommerziellen Spielzeugläden (Imfeld 2012). Die Zuschreibungen von Spielzeug und gender haben sich wenig verändert. Die Studie von Dannhauer aus dem Jahr 1977 verweist darauf, dass Spielsachen sehr geschlechtsspezifisch konnotiert sind. Die Befragten waren sich generell einig, was ein Jungen- und was ein Mädchenspielzeug sei. Diese Studie wurde 2008 repliziert (Andrey und Nyffenegger 2008; Grünewald-Huber 2009). Es zeigte sich, dass die geschlechterstereotype Zuschreibung fast unverändert fortbesteht. Zudem wird angenommen, dass die geschlechterdifferente Vermarktung von Spielwaren seitdem zugenommen hat (Imfeld 2012).

Im Kontext des Forschungsprojekts stellt sich die Frage, welche Möglichkeiten für doing und undoing gender in den räumlichen Arrangements der Kitas bereitgestellt werden und auf welche Weisen der geplante Raum mit seinen Ordnungsstrukturen und Repräsentationen doing oder aber undoing gender vermittelt und nahelegt.

\subsection{Ethnografische Raumanalyse}

Um zu untersuchen, wie sich doing und undoing gender im Raum der Kitas zeigt, eignet sich innerhalb der ethnografischen Forschungsstrategie die Erhebung von visuellen Daten (Pink 2008). Die Gestaltung der Kitaräume wurde in einer Raumbegehung von der Kitaleitung gezeigt und erläutert und von den Forschenden strukturiert durch Fotos und Raumskizzen dokumentiert und analysiert. Die visuellen Daten wurden mit Feldnotizen und Audioaufnahmen ergänzt. 


\subsubsection{Datenerhebung: Raumbegehung, Fotos und Raumskizzen}

Die Raumbegehung wurde in 20 Deutschschweizer Kitas durchgeführt. Nachdem die Kitaleitungen für die Teilnahme am Forschungsprojekt zugesagt hatten, wurde ein Besuch mit Interview mit der Kitaleitung und Raumbegehung vereinbart, mehrheitlich außerhalb der Öffnungszeiten. Bei der Raumbegehung forderten wir die Kitaleitung dazu auf, uns die Räume nicht nur zu zeigen (räumliche Praktiken), sondern auch zu erzählen, wie sie jeweils genutzt werden (gelebter Raum) und welche konzeptionellen Überlegungen damit verbunden sind (geplanter Raum). Die Erläuterungen der Kitaleitung wurden in Protokollen und mit Audioaufnahmen festgehalten. Indem die Kitaleitung durch die Räumlichkeiten der Kita führt, findet ein place making (Pink 2008) statt, das mit den Forschenden geteilt wird. Die Raumbegehung wurde bewusst zeitlich so vereinbart, dass keine Kinder mehr anwesend waren. Die Dinge und Bereiche sind in einer aufgeräumten Kita viel sichtbarer so angeordnet, wie es von den Fachpersonen gedacht und vorgesehen ist. Dies ermöglicht es noch besser, die Strukturierung des Raums durch die Fachpersonen erkennen zu können und somit den geplanten Raum und die darin enthaltenen Raumrepräsentationen analysierbar zu machen.

Mittels Fotografie wurden alle Räume systematisch dokumentiert. In jedem Raum wurden vier Fotos in alle Richtungen aufgenommen. Dies entspricht innerhalb der visuellen Ethnografie einem ,photographic survey“ (fotografische Überblickserhebung) (Pink 2007, S. 74 f.). Zusätzlich wurden einzelne Details fotografiert, dies mit Fokus auf Spielmaterialien (zum Beispiel Verkleidungsecken) und Ordnungsstrukturen (zum Beispiel Garderobe). Dieses Vorgehen wurde zwischenzeitlich auch von anderen Forschenden zu Raum und Kita verwendet, so beispielsweise von Knauf $(2017,2019)$.

Die Forschenden skizzierten während des Besuchs oder direkt im Anschluss daran die Anordnung der Räume sowie innerhalb der Räume die wichtigsten Bereiche als Plan (Abb. 3.1). Wichtig war dabei die grobe Anordnung, es wurden keine Vermessungen vorgenommen. Das Vorgehen orientiert sich am ethnografischen Vorgehen, bei dem Raum in die Analysen einbezogen wurden, beispielsweise Breidenstein und Kelle (1998), Jung (2009) oder Kuhn (2013).

Das Datenmaterial für die Raumanalyse umfasste 858 Fotos aus 20 Kitas, durchschnittlich 43 pro Kita, sowie je eine Raumskizze pro Kita. Zu beachten ist, dass Fotos ebenso wie Feldnotizen und Videoaufnahmen durch die Forschenden mitbeeinflusste Datenerhebungsverfahren darstellen. Das Foto zeigt immer einen Ausschnitt, die Datensammlung mit der Kamera fokussiert auf einen Gegenstand und beachtet andere nicht (Pink 2008). Mit dem hier vorgestellten systematischen 

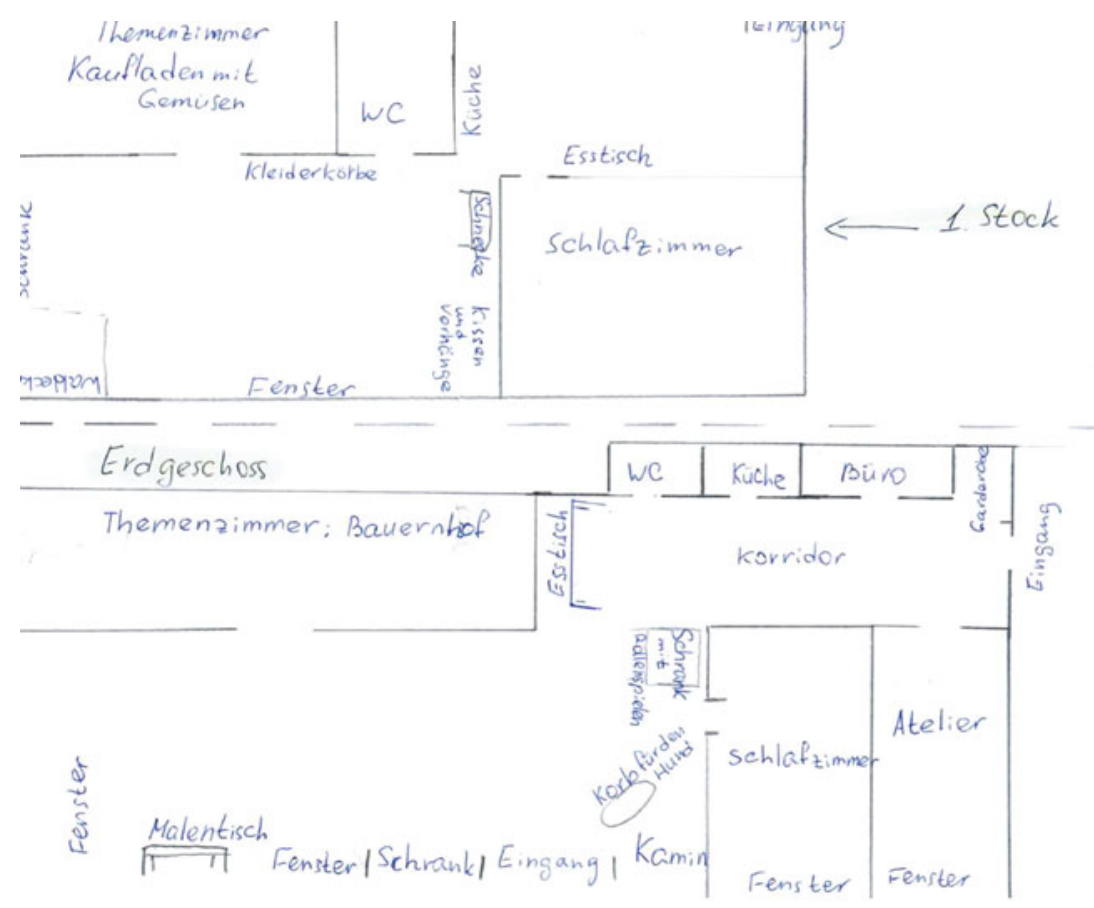

Abb.3.1 Beispiel Raumskizze

Vorgehen reflektieren wir dieses Spannungsfeld, indem wir die durch die Fotografien und Raumskizzen eingenommenen Blickrichtungen systematisierten. Wichtig für die Auswahl der Details war zudem der fortlaufende Vergleich zwischen den Kitas. Durch ein kontinuierliches Vergleichen entsteht im ethnografischen Forschungsprozess (Vogt 2002) Aufmerksamkeit auf bestimmte Details der Organisationskultur, die für die Analyse von doing und undoing gender in der Kita besonders relevant sein können.

\subsubsection{Datenanalyse: Kategorienbildung und Interpretation}

Die Fotos, die jeden Raum aus allen vier Richtungen abbilden, sowie die Fotos von Einrichtungsdetails, die den Forschenden interessant erschienen, bilden 
Tab. 3.1 Kategorisierung visuelle Inhaltsanalyse der Bereiche und Spielmaterialien, Ausschnitt zur Illustration $(1=$ vorhanden $)$

\begin{tabular}{l|l|l|l}
\hline Kita & Bauecke & Auto, Garage & Ort \\
\hline 4 & 1 & & \\
\hline 7 & 1 & 1 & Lego bei Bauklötzen, Zug bei Puppen \\
\hline 19 & 1 & 1 & Lego und Garage zusammen \\
\hline$\ldots$ & $\ldots$ & $\ldots$ & \\
\hline Total $(\mathrm{n}=20)$ & 20 & 15 & \\
\hline
\end{tabular}

Aspekte des Raums und der Materialien ab, die als Teil des ethnografischen Vorgehens reflektiert und interpretiert werden müssen (Pink 2007). Die Datenanalyse umfasst zwei Ansätze: Erstens die Analyse der vorhandenen Spielangebote und ihrer räumlichen Anordnung durch die Entwicklung von Kategorien, die tabellarisch als Überblick dargestellt wurde; zweitens wurden Fotos von Spielbereichen im Detail in Forschungswerkstätten gemeinsam interpretiert.

Für einen Überblick über die 20 Kitaräume wurde mithilfe der Fotos und der Raumskizzen für jede Kita analysiert, was räumlich vorhanden ist und in welcher räumlichen Nähe diese Bereiche und Materialien zueinander angeordnet sind. Die Kategorien der räumlich definierten Bereiche und Materialien wurden inhaltsanalytisch (Kuckartz 2012) aus den Fotos und Raumskizzen entwickelt. Aufgrund der Fotos wurde für jede Kita entschieden, ob räumlich definierte Bereiche für diese Kategorien sichtbar sind. Zudem wurde festgehalten, welche Bereiche und Materialien in räumlicher Nähe zueinanderstehen. Ein Ausschnitt dieser Kategorisierung ist in Tab. 3.1 für Autos und Bauecken für drei der 20 Kitas beispielhaft aufgeführt.

In einem zweiten Schritt wurden die Fotos der jeweiligen Bereiche genauer betrachtet und in gemeinsamen Forschungswerkstätten im Forschungsteam wie auch mit Studierenden hinsichtlich der bestehenden Genderismen interpretiert. Als Deutungsrahmen wurden hier Untersuchungen zu stereotypen Ordnungen von Spielzeugen herangezogen (Dannhauer 1977). Zudem haben Studierende der Pädagogischen Hochschule St.Gallen einen Fragebogen ausgefüllt, in dem sie Spielzeuge als männlich oder weiblich konnotiert oder neutral einschätzten. Diese historischen wie auch aktuell erhobenen Einschätzungen sowie die Beschreibung von Genderismen aus der Forschungsliteratur und die Forschung zu Geschlechterstereotypen (Eckes 2008) wurden als Grundlage für die Analyse der Häufigkeiten und der räumlichen Anordnungen wie auch für die interpretative 
Analyse von Detailaufnahmen verwendet. Die Zuordnungen dienen als Arbeitsinstrument für die Beschreibung von doing und undoing gender im räumlichen Arrangement.

Diese Analysen wurden für Bereiche gemacht, die in vielen Kitas vorkamen: Großes Rollenspiel, kleines Rollenspiel sowie Gestaltung. Dabei kommt der Zugang der visuellen Ethnografie (Pink 2008) zum Tragen. Wie Pink (2008) erläutert, ist Ethnografie eng mit der Konstruktion von Räumen verbunden: Sie untersucht, wie Teilnehmende ihren Raum schaffen, wie durch die Darstellung der Forschung Raum wiederum konstituiert wird.

\subsection{Doing und undoing gender in der räumlichen Gestaltung der Kita}

Aus der auf Grundlage der Raumskizzen und Fotos erstellten Raumanalyse werden zunächst die Ergebnisse der visuelle Inhaltsanalyse dargestellt, um die räumlichen Strukturen der Kitas darzustellen und auf doing und undoing gender hin zu beschreiben. Anschließend wird für eine vertiefende Analyse des doing und undoing gender in diesem Kapitel der Bereich des großen Rollenspiels ausgewählt. Dabei werden die Fotos detailliert in Bezug auf doing und undoing gender analysiert und die Protokolle zur Raumbegehung mit den Erläuterungen der Kitaleitungen einbezogen.

\subsubsection{Genderismen in den räumlichen Arrangements}

In Tab. 3.2 findet sich die Analyse der Häufigkeiten der räumlich definierten thematischen Bereiche in den Innenräumen der 20 untersuchten Kitas. Die erste Spalte führt die Bereiche und Angebote auf, die häufig als räumliche Einheiten eingerichtet sind. Dies ist eine datenbasierte Kategorisierung aufgrund der visuellen Inhaltsanalyse der Raumskizzen und Fotos. Es wird angegeben, in wie vielen Kitas diese Ecken und Angebote vorkamen.

Die Bereiche werden weiter den Bildungsbereichen der Curricula und/oder der Spielkategorie zugeordnet. Die Unterteilung der Spielarten nimmt Definitionen des Spiels (Hauser 2016) auf. Unterschieden werden hier das Rollenspiel (auch Fantasiespiel und So-tun-als-ob-Spiel genannt), unterteilt in großes Rollenspiel (die Kinder sind selbst die imaginäre Figur) und kleines Rollenspiel mit Figuren, weiter das Bewegungsspiel, das Konstruktionsspiel sowie gestalterische Aktivitäten. Den Angeboten wurden die Bildungsbereiche zugewiesen, wie sie 


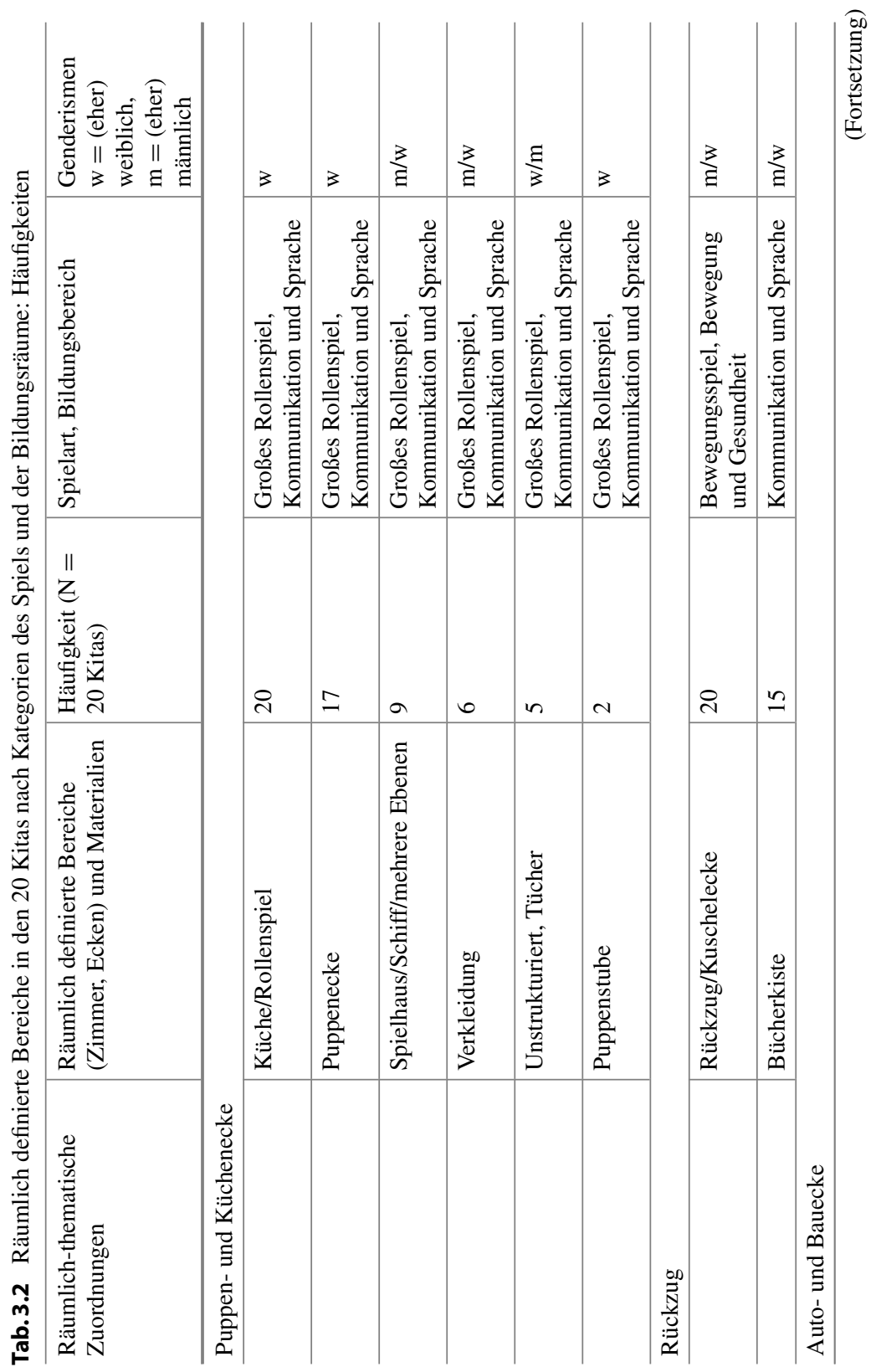




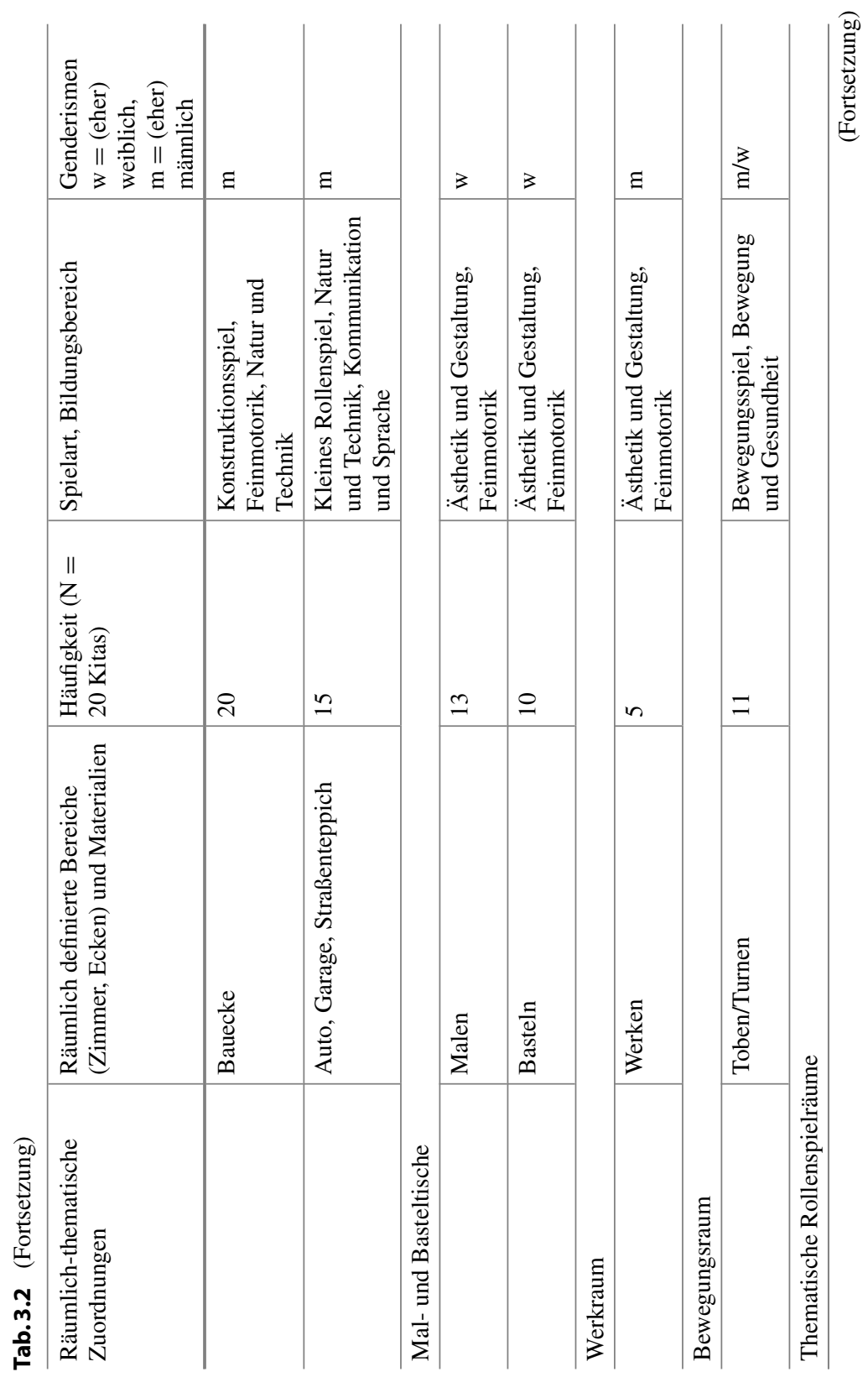




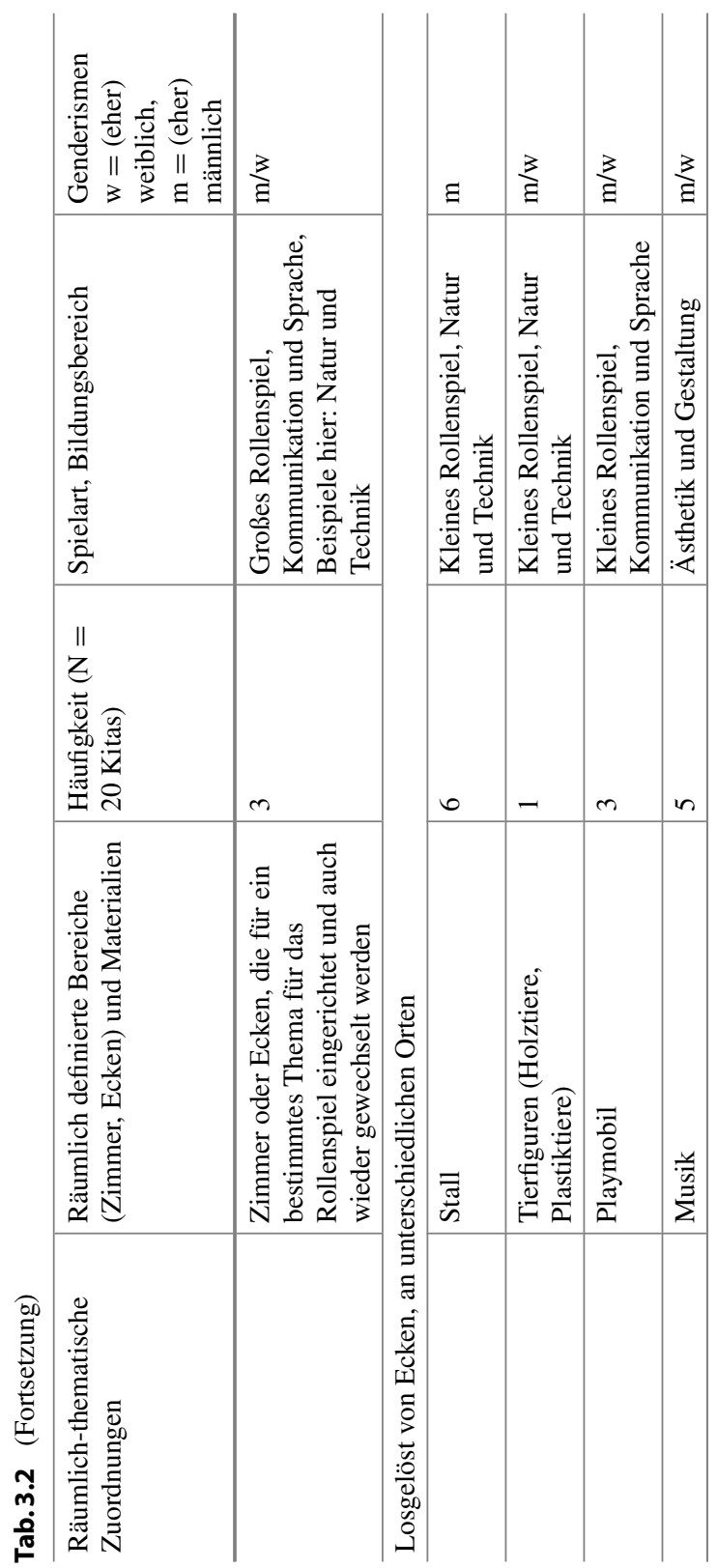


in Curricula für den Frühbereich häufig definiert werden, beispielsweise in den Erfahrungsfeldern der Stadt Zürich (Walter-Laager 2015) oder im österreichischen BildungsRahmenPlan (Ämter der Landesregierungen der österreichischen Bundesländer 2009).

Die Tabelle wurde weiter mit einer Zuordnung zu weiblich oder männlich konnotierten Tätigkeiten, Spielsachen und Lebensbereichen ergänzt, im Sinn von Genderismen (Goffman 1994). So sind Haushalt, Küche und Kinderbetreuung weiblich konnotiert, Autos, Bauklötze und Stall männlich. Im Bereich von Ästhetik und Gestaltung sind Malen und mehr noch Basteln als weibliche Tätigkeit assoziiert, Werken als männlich, Musik als männlich und weiblich. Einzelne Angebote wurden beiden Geschlechtern zugeteilt.

Die Ergebnisse dieser Analyse sind in Tab. 3.2 dargestellt.

Die in Tab. 3.2 dargestellten Raumstrukturen lassen sich nach Ordnungsprinzipien untersuchen: Welche Aspekte werden berücksichtigt, wenn eine räumliche Ordnung hergestellt und entschieden wird; welche Spielmaterialien werden auf welche Weise zusammengefasst? Der Vergleich der räumlichen Zuordnung, der pädagogischen Kategorisierung von Spiel und Bildungsbereich und der Genderismen zeigt deutlich, dass die gewählte thematische Zuordnung den Genderismen folgt, jedoch die Kategorien nach Art des Spiels oder Bildungsbereichs häufig nicht berücksichtigt. Deutlich ist das Ordnungsprinzip der Genderismen im Spielbereich des kleinen Rollenspiels und im Bildungsbereich der Ästhetik und Gestaltung zu erkennen.

Zum kleinen Rollenspiel, auch Kleine-Welt-Spiele genannt, gehören Puppenstube wie auch Playmobil, Stall, Autos und Garage und ähnliches (Sarbach 2011). Bei dieser Spielart bewegen die Kinder eine Figur, sprechen für sie und entwickeln Spielnarrative. Die Puppenstube des kleinen Rollenspiels, die weiblich konnotiert ist, wird in der Nähe der ebenfalls weiblich konnotierten Küchenund Puppenecke aufgestellt. Autos, Garagen und Straßenteppich, ebenfalls kleines Rollenspiel, bilden hingegen Spielecken, in denen kaum Figuren vorkommen. Oft werden sie mit Bauklötzen und Lego kombiniert, die ebenfalls männlich konnotiert sind. Diese räumliche Anordnung folgt der geschlechterstereotypen Zuteilung des Themas. In der Raumordnung wird so doing gender dramatisiert, während die Spielart des kleinen Rollenspiels nicht als Ordnungsprinzip berücksichtigt wird. In der räumlichen Anordnung der Themenbereiche und Spielmaterialien zeigt sich zudem ein in den Raum eingeschriebener Genderismus: Die Aufteilung in weiblich konnotiertes kleines Rollenspiel und männlich konnotiertes Rollenspiel schreibt eine Trennung von öffentlicher und privater Sphäre fort. Während Autos, Bauklötze und Straßenteppich eine Spielecke bilden, die das Spielen im imaginären öffentlichen Raum nahelegt, steht die Puppenstube neben der Küchen- 
und Puppenecke; damit wird ihre Zugehörigkeit zur privaten Sphäre betont. Auf diese Weise wiederholt sich die damit verbundene Zuweisung der Privatsphäre als weiblicher und der Arbeitswelt als männlicher Domäne.

Das doing gender in der räumlichen Anordnung transportiert nicht nur Genderismen (weiblich-häuslich; männlich-öffentlich) sondern erschwert auch verbindende Spielnarrative, bei denen beide Domänen integriert werden. Durch die räumlich getrennte Anordnung von Puppenstube und Straßenteppich wird es den Kindern erschwert, diese Themenbereiche im Spiel zu verbinden. Wie andernorts ausgeführt, erschwert die räumliche Anordnung, dass die Kinder Spielnarrative ihres Alltags im kleinen Rollenspiel auszudrücken, beispielsweise aufstehen und frühstücken zu Hause, mit dem Auto zur Kita gefahren werden, Berufstätigkeit der Eltern in der Stadt und Rückkehr zu Kita und nach Hause. Dafür müsste die Puppenstube zum Straßenteppich und den Autos gestellt werden (Vogt et al. 2013).

Auch in den Bildungsangeboten des Bereichs Ästhetik und Gestaltung zeigt sich ein doing gender in der räumlichen Anordnung: Die männlich konnotierten Werkräume befinden sich oft weiter weg von den hauptsächlichen Gruppenräumen, häufig im Keller. Die weiblich konnotierten Bastelmaterialien mit Farben, Glitzer, Papier, Fäden, Stoffen etc. finden sich hingegen oft im Kitagruppenraum und sind so besser zugänglich. Allenfalls wird - häufig ebenfalls im Keller, aber getrennt vom Werkbereich - ein Malatelier eingerichtet. Auch wenn alle diese Angebote zum Bildungsbereich Ästhetik und Gestaltung gehören, werden Malen und Basteln sowie Werken voneinander räumlich getrennt. Die Trennung folgt der stereotypen Zuordnung von Malen und Basteln - häufig in ,Glitzerästhetik“ (Tennhoff et al. 2014) - als weiblich konnotiert und Werken als männlich konnotiert.

In der Strukturierung der Kitaräume mit der Definition von Ecken, Spielbereichen und in der Anordnung der Materialien ist ein doing gender angelegt. Angebote werden thematisch nach Genderismen angeordnet; andere mögliche Ordnungsmuster werden nicht berücksichtigt, die beispielsweise den Spielarten (zum Beispiel kleines Rollenspiel) oder auch den Bildungsbereichen (zum Beispiel Ästhetik und Gestaltung) folgen. Die räumliche Anordnung folgt hier Traditionen, die Geschlechterordnung ist in der Materialität und in den räumlichen Ordnungsprinzipien eingeschrieben, wie dies auch in den Materiallisten für Kindergartenausstattung (Sarbach 2011) oder in den Marketingstrategien in Spielwarenhandlungen geschieht (Imfeld 2012).

In einzelnen Kitas wird diese räumliche Trennung unterlaufen, was wir als räumliches undoing gender interpretiert haben. Zum Beispiel wurden in einer Kita das Malen und Werken zusammengelegt. Das Foto (Abb. 3.2) zeigt Farbstifte, 


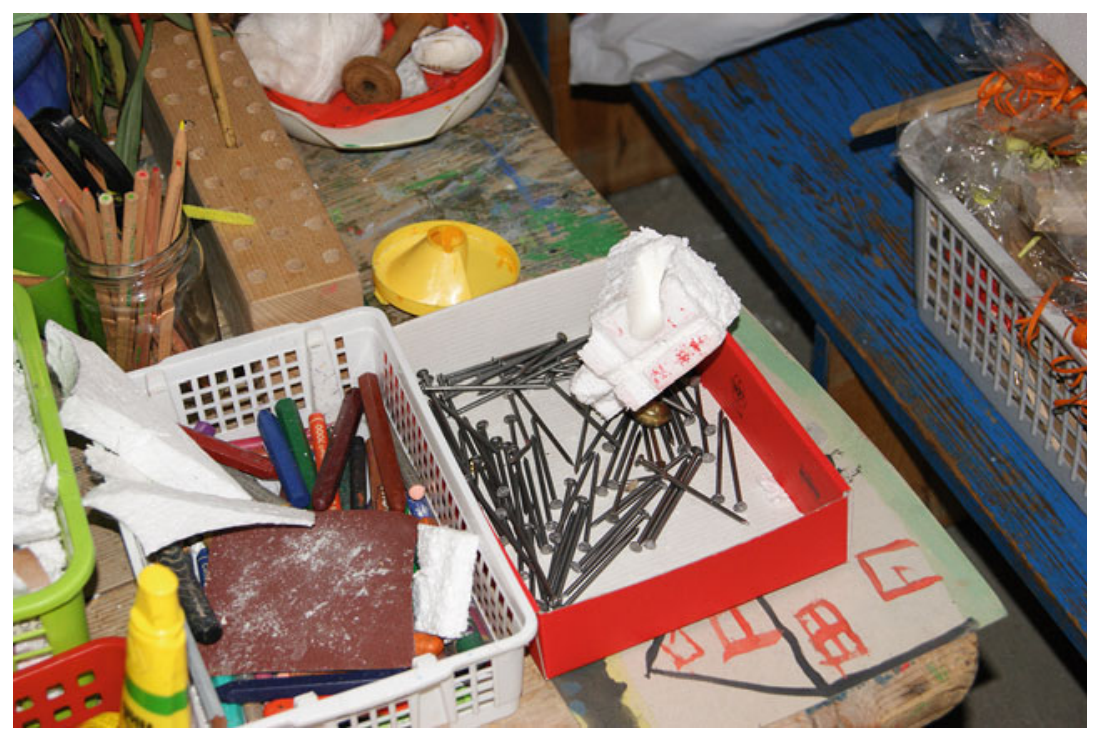

Abb. 3.2 Werken, Malen und Basteln in einer Kiste - undoing gender oder Unordnung?

Nägel, Abfallmaterialien, Schmirgelpapier nebeneinander, teilweise in der gleichen Schachtel. Diese Aufnahme ist als Ausnahme von der häufig beobachteten Regel interessant. Wir haben die Aufnahme in mehreren Transferworkshops mit Praktikerinnen und Praktikern zur Diskussion gestellt und als ein - von uns aus positiv interpretiertes - Beispiel, wie doing gender durch die räumliche Anordnung im Bereich Gestalten aufgehoben werden kann, gerahmt. Das Foto löste dabei häufig negative Reaktionen aus: Dass Nägel, Schmirgelpapier und Farbstifte beieinander aufbewahrt und gerade nicht in räumlich voneinander getrennten Bereichen aufgeräumt wurden, wurde als Unordnung wahrgenommen.

\subsubsection{Das große Rollenspiel: über Puppen und Küche hinaus?}

Eine Puppen-Familien-Küchen-Rollenspielecke ist in allen besuchten Kitaräumen zu finden. Dies ist ein häuslich-privater Tätigkeitsbereich, die traditionelle und weit verbreitete Arbeitsteilung (BfS 2017; Moreno-Colom 2017) macht ihn zu einem weiblich konnotierten Bereich. Die Einrichtungsgegenstände und farbliche Gestaltung der Puppen- und Küchenecke verstärkt in einzelnen Kitas die 
Kennzeichnung als weiblicher Bereich. In fünf der 20 Kitas ist die Küchenecke als weiblicher Ort gekennzeichnet, beispielsweise in Rosa gehalten (5/20). Mehrheitlich sind als Farbgebung Rot und Holzfarben zu finden.

Bei allen Puppen- und Küchenecken gehören ein Tisch mit Stühlen zum Essen, ein Spielkochherd und Spielgeschirr dazu. Einzelne haben auch eine Spüle sowie Küchen- und Haushaltsgeräte, wie ein Spielzeugbügeleisen, Staubsauger, Mixer und ähnliches. Viele Küchenecken sind gleichzeitig die Bereiche der Puppen, mit Puppenbettchen, -wiegen und -kinderwagen (17/20). Auch hier dominiert in einigen Kitas die rosa Farbgebung. Die Puppen selbst lassen sich aufgrund der Fotos nicht nach ihrer Geschlechtszuordnung oder ethnischen Diversität bestimmen.

Ein Beispiel für die rosa Farbgebung ist auf Abb. 3.3 zu sehen. Die weibliche Konnotation des Rollenspielbereichs ist sichtbar im rosa Schminktisch, der mit Blümchen und Glitzer dekoriert ist, sowie den Tülltüchern, die von der Decke hängen. Im Rollenspielbereich ist rechts auch ein großes rotes Schiff, das in Bezug auf gender männliche und weibliche Aspekte repräsentiert. Bei der Raumbegehung fällt den Forschenden das rote Telefon auf dem rosa Schminktisch auf. Auf Nachfrage berichtet die Kitaleitung, dass die Kinder im Rollenspielbereich Feuerwehr gespielt haben. Der Schminktisch war die Telefonzentrale der Feuerwehr.

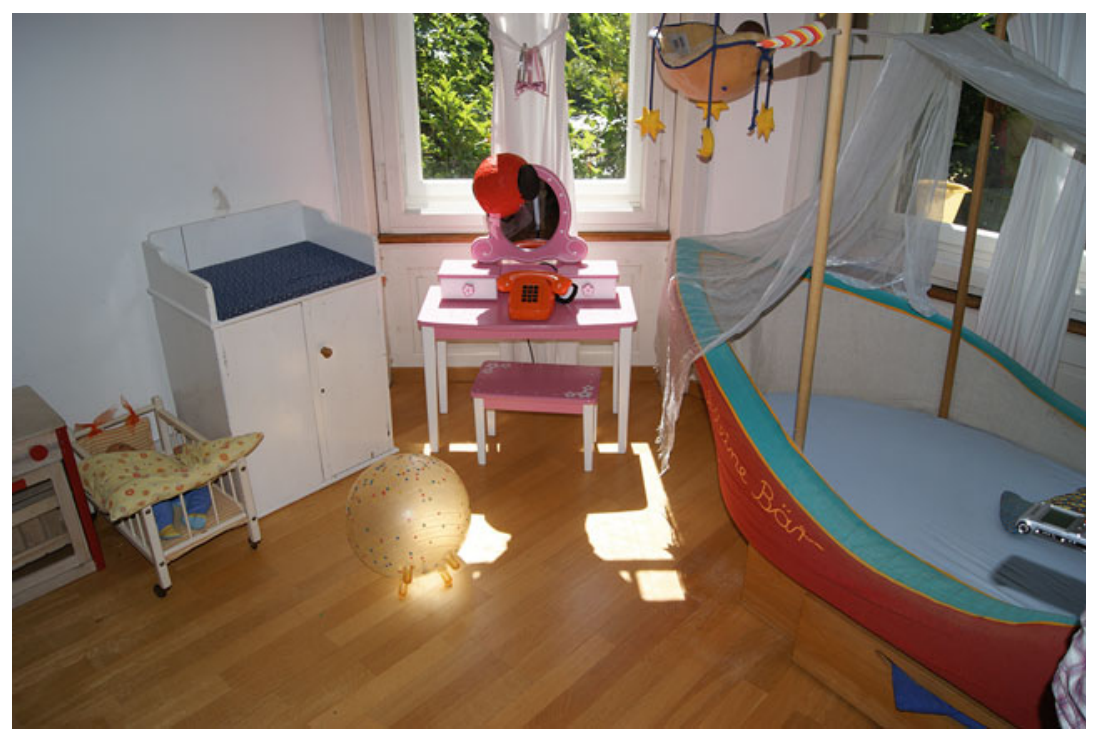

Abb. 3.3 Rosa Schminktisch, verziert mit Blümchen und Glitzer, darauf ein rotes Telefon 
Über dem Schminkspiegel hängt darum auch noch der rote Feuerwehrhelm. Die Kinder deuteten hier die weiblich angelegte Konnotation des Bereichs um, was als undoing gender interpretiert werden kann. Beim Aufräumen am Abend wurde dieses undoing gender im Raum gelassen und nicht wieder in die Aufräumordnung und die räumliche Ordnung des doing gender zurückgesetzt. Solche Spuren von gelebtem Raum, die die Ordnung durchbrechen und als räumliches undoing gender gesehen werden können, waren nur in wenigen Kitas zu sehen.

Häufig sind auch Verkleidungen und Requisiten in den Küchenecken oder in unmittelbarer Nähe dazu zu finden. Nicht in allen Kitas konnte systematisch analysiert werden, welche Verkleidungen und Requisiten zur Verfügung stehen. In sechs Kitas sind diese jedoch auf den Fotos gut erkennbar, so beispielsweise in Abb. 3.4 und 3.5.

In der einen Kita wurden zur Verkleidung Hüte, Schmuck, Schuhe, Taschen und Sonnenbrillen bereitgestellt (Abb. 3.4). Alle vorhandenen Hüte, Schuhe, Handtaschen und der Schmuck sind Damenhüte, Damenschuhe, Damenhandtaschen und Damenhalsketten, die Sonnenbrillen würden für Damen und Herren passen.

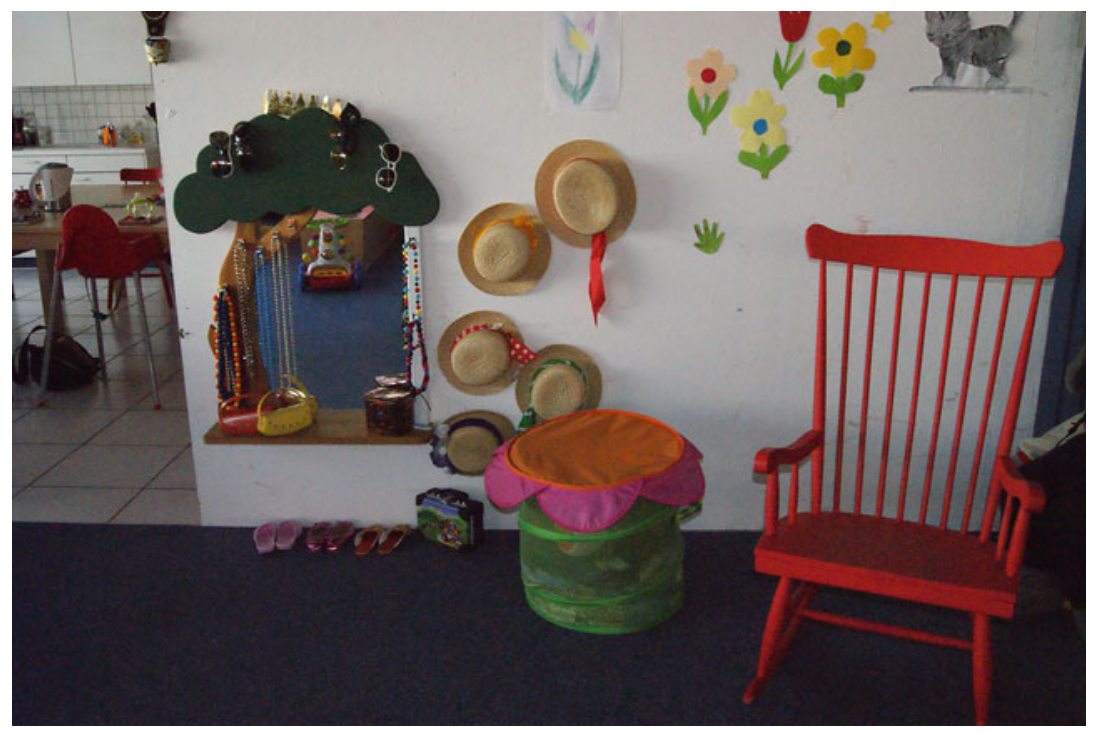

Abb. 3.4 Requisiten für das große Rollenspiel: Damenschuhe, Koffer, Damenhandtaschen, Halsketten, Sonnenbrillen, Krone und Damenhüte 


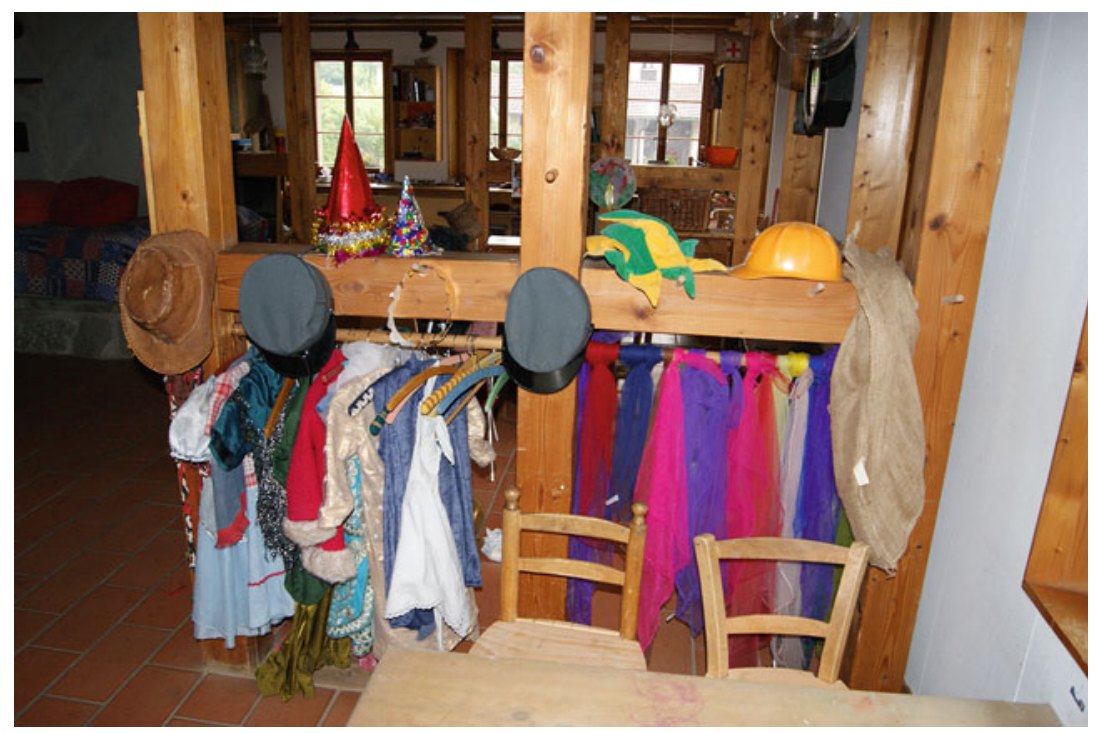

Abb.3.5 Verkleidung und Requisiten für männlich und weiblich konnotierte Rollen sowie Tücher als unstrukturiertes Material

Anders in einer anderen Kita, in der Hüte, Verkleidungen und Tücher bereitgestellt wurden (Abb. 3.5). Die Hüte sind Funktionen und Berufen zugeordnet, die stärker männlich konnotiert sind, wie Bauschutzhelm, Polizei, Ranger und Narr oder wenig definiert sind, wie die spitzen Hüte. Die Verkleidungen und Requisiten sind hier variantenreicher, sie reichen vom Prinzessinnenkleid in Tüll und Rosa zu einem Bauarbeiteroverall. Es stehen zudem auch verschiedenfarbige Tücher zur Verfügung, die für das Verkleiden genutzt werden können. Diese Kita hat als einzige der Kitas Verkleidungen, Requisiten und unstrukturierte Verkleidungsmaterialien zusammen angeboten, während von den 20 Kitas fünf Kitas Verkleidungsecken eingerichtet haben und vier unstrukturierte Verkleidungsmaterialien bereitstellten.

Mit den Verkleidungen und Requisiten in der Rollenspielecke werden den Kindern Rollen für das Fantasiespiel zur Verfügung gestellt und damit auch nahegelegt. Wenn die Rollenspielverkleidungen in der Nähe der Küchenecke angeordnet sind und fast ausschließlich weibliche Kleidungen enthalten, wird ein doing gender in den Raum eingeschrieben: Küche und Familie sind Bereiche der Frauen. Damit wird durch das Angebot von Requisiten und die räumliche 
Anordnung für das So-tun-als-ob-Spiel die traditionelle Rolle einer Mutter, die für Haushalt und Kinderbetreuung zuständig ist, suggeriert (Tennhoff et al. 2014). Damit ist nicht ausgeschlossen, dass Kinder in der Performanz des Spiels ein undoing gender zeigen, aber die geschlechterstereotype Arbeitsteilung wird über die vorhandenen Requisiten als räumliche Praxis angeboten und damit normativ wahrgenommen.

Methodisch entscheidend ist nicht nur das Erfassen dessen, was da ist, und die Analyse der Genderismen dieses Materials, sondern auch die Reflexion dessen, was fehlt. Diese Lücken sind ebenfalls zentral für die Analyse vor dem Hintergrund der Schweigsamkeit des Sozialen (Hirschauer 2006). Dramatisierung von Geschlecht erfolgt nicht nur über das, was getan wird, sondern auch über das Unterlassen, also das, was nicht getan wird. In fast allen Puppen- und Küchenecken fehlen männlich konnotierte Kleidungsstücke, Schuhe und Requisiten (zum Beispiel Krawatten). In Bezug auf die häuslichen Tätigkeiten, die mit Spielgegenständen gespielt werden können, finden sich wenig handwerkliche Spielmaterialien wie Werkzeugkästen und Spielzeugbohrer und es sind auch keine Bürogegenstände, wie beispielsweise ein Pult mit Computer, zu sehen. Auch hier ist ein doing gender im Raum zu konstatieren: Eine Fokussierung auf die traditionell weiblichen Bereiche im Haushalt verbunden mit einer klaren Trennung von Tätigkeiten in der Familie und in Berufen außerhalb.

Die Kinder und die Kinderbetreuenden nutzen das Raumangebot und die Spielmaterialien für ihre je eigenen Interpretationen im Spiel. Sie können im Rollenspiel die Verkleidung der Polizei den Jungen und das Tüllkleid den Mädchen zuweisen, und damit doing gender zeigen oder undoing gender in der Interaktion realisieren und nicht geschlechterstereotyp verteilen (Kap. 4). Im Spiel selbst könnte die Spielhandlung auch mit geschlechterstereotyper Verteilung der Verkleidungen geschlechteruntypisch interpretiert werden, wenn die Prinzessin im Tüllkleid die Kriminalität bekämpft und der Polizist für die Familie kocht, was wiederum einem undoing gender im Sinn der Umdeutung von Geschlechterstereotypen entsprechen würde.

Für das große Rollenspiel wurden nur in drei Kitas andere Themen eingebracht: Eine Kita hat zum Thema Bauernhof ein Bauernhof-Rollenspielzimmer eingerichtet, in dem Strohballen, ein Kübel, ein Schaukelpferd und ein kleiner Bauernhof mit Holzkühen lagen. Eine weitere Kita hatte ein BaustellenRollenspielzimmer mit leeren Farbkübeln, Signalhütchen, Lastwagen, Bagger und Schutzhelmen eingerichtet. Eine weitere Kita hatte Häuser aus Karton und Tüchern in einer Ecke, auf dem Foto sind keine weiteren thematischen Spielgegenstände dazu zu erkennen. Dass nur so selten weitere Themen in den Rollenspielbereichen der 20 besuchten Kita zu sehen waren, wirft Fragen auf. 
Gemäß Bildungsplänen für den Kindergarten ist die anregende Lernumgebung für das freie Spiel entscheidend. So schreiben Wustmann Seiler und Simoni (2012, S. 27) im „Orientierungsrahmen für frühkindliche Bildung, Betreuung und Erziehung in der Schweiz“: „Kinder müssen Themen und Lernräumen begegnen, die sie mit neuen, interessanten Inhalten herausfordern und die sie mit ihren bisherigen Erfahrungen verbinden können“. Die nahezu ausschließliche Fokussierung auf die häusliche Welt mit der in allen 20 Kitas vorhandenen Puppen- und Küchenecke, die zudem in 17 der Kitas durch keine weiteren Spielsituationen oder Themen für das große Rollenspiel ergänzt werden, muss als räumliches doing gender in der Kita interpretiert werden. Die Rollenspielbereiche, die traditionell zur Einrichtung von Kitas und auch Kindergärten gehören, gehören in den Bereich des Privaten und sind weiblich konnotiert. Die fixen, raumeinnehmenden Rollenspielbereiche zeigen ein doing gender, ein doingfeminity, bzw. ein doing housewife feminity (Nentwich et al. 2016; vgl. Kap. 5). Rollenspiele aus anderen Kontexten, wie zum Beispiel Requisiten zur Darstellung verschiedener Berufe, nehmen räumlich kaum Platz ein und weitere, im modernen Alltag relevante Themen wie Flughafen, Bahnhof, Fabrik, Handy-Reparaturstätten, Spitäler, Tierarztpraxen und vieles mehr, sind in der Kita nicht vorhanden. Über die räumliche Gestaltung der Kita in der Aufteilung in unterschiedlich geschlechtlich konnotierte Bereiche und die damit verknüpften räumlichen Praktiken findet in erster Linie ein doing gender statt. Ein undoing gender ist bis auf wenige Ausnahmen dem gelebten Raum und den darin stattfindenden Interaktionen überlassen.

\subsection{Potenziale der Raumanalyse als Zugang zu (un)doing gender}

Fotos als visuelle Daten eignen sich für die Wissenskonstruktion in einer ethnografischen Forschungsstrategie sehr gut. Zugleich ist es wichtig, visuelle Daten und ihre Analyse kritisch zu reflektieren (Pink 2008). Fotos sind genauso wie Feldnotizen und Videoaufnahmen durch die Forschenden mitbeeinflusste Datenerhebungen. Das Foto zeigt immer einen Ausschnitt, die Datensammlung mit der Kamera fokussiert auf einen Gegenstand und beachtet andere nicht. Im hier vorgestellten Vorgehen wird dieses Spannungsfeld durch drei Zugangsweisen reflektiert: Erstens wurden in jedem Raum in jede der vier, durch die meist viereckige Raumform vorgegebenen Blickrichtungen Fotos gemacht. Zweitens wurde auf der Basis des Rundgangs und der Fotos eine Raumskizze für die räumliche Anordnung gezeichnet. Drittens wurden induktiv, auf Grundlage der Forschungsfragen des Projekts wie auch im laufenden Vergleich zwischen den Kitas, Details 
von den Forschenden ausgewählt und fotografiert, die eine Kita im Vergleich zu anderen Kitas auszeichneten oder die für doing und undoing gender besonders relevant schienen. Der Einbezug des Raums als entscheidende Zugangsweise für Organisations- und Bildungsforschung, was als spatial turn referenziert wird (Gulson und Symes 2007), weist großes Potenzial auf, das noch nicht ausgeschöpft ist.

Der Raum wurde von der Kitaleitung gezeigt und damit gedeutet; die Fotos machten die Forschenden. Die Perspektive der Kinder auf den geplanten Raum oder ihre Nutzung des gelebten Raums wurde hier nicht einbezogen. Dieser Fokus ist für die Erforschung von doing und undoing gender in der Kita als Institution sinnvoll. Im Zentrum unserer Analyse steht die Kita als gendered organisation (Acker 1990). Dafür sollen die Raumstrukturen, wie sie von den Fachpersonen geplant und eingerichtet wurden, im Zentrum stehen. Der aufgeräumte Kitaraum zeigt die Ordnungsstrukturen. Diese Ordnungsstruktur orientiert sich, wie in den Ergebnissen dargestellt wurde, an den Genderismen als Ordnungsprinzipien; es wurde mehrheitlich doing gender in den räumlichen Arrangements festgestellt, und selten räumliches undoing gender.

Für eine weiterführende pädagogische Theoretisierung wie auch für eine soziologisch und psychologische Interpretation der Bedeutung der institutionellen Gestaltung für das Aufwachsen von Kindern wäre es wichtig, die Interpretation der Kinder einzubeziehen. Mit Kindern im Grundschulalter sind Raumführungen und Fotografien der Räume durch die Kinder geeignete Zugänge (Fritzsche et al. 2011), ebenso mit Kindern im Schulalter in einer ethnografischen Forschung zu Spielplätzen (Karsten 2003). Für Vorschulkinder müssten Forschungszugänge noch entwickelt werden, die sich möglicherweise auf die Bewegung und das Handeln der Kinder im Raum stützen könnten. Für das hier dargestellt Forschungsvorhaben, doing und undoing gender in der Kita zu analysieren, erwies sich der Fokus auf den geplanten Raum, ersichtlich an den nach den vorgesehenen Ordnungsstrukturen aufgeräumten Kitas, als sehr geeignet.

Die aufgeräumten Kitas und der Fokus auf die von den Kinderbetreuenden organisierten Raumstrukturen sind ein wichtiger Analysefokus, der in der Forschungsliteratur bisher wenig beachtet wird. In einer Sichtung der Forschung zu Schulräumen ist gerade dieser Fokus, der eine Mesoebene darstellt, noch zu wenig erforscht. Forschung zur Schulhausarchitektur fokussiert auf Raumstrukturen, die außerhalb des Einflussbereichs der pädagogischen Fachpersonen liegt. Ethnografische Forschung zu Ordnungsstrukturen fokussiert auf dem sozialen, gelebten Raum und beschreibt mikroanalytisch Interaktionen im Raum, beispielsweise Jung (2009), Kuhn (2013) oder Kubandt (2016). Die Praxisliteratur orientiert 
sich häufig an den pädagogischen Traditionen, die im Raum ihren Ausdruck finden, wie Montessori, Reggio Emilia und weiteren. Für die Forschungsfrage des doing und undoing gender in der Kita ist der Fokus auf die räumliche Anordnung der Spielangebote und die Materialisierung als Mesoebene zielführend, weil dadurch beide raumsoziologischen Aspekte des Raums, seine Strukturierungsmacht wie auch seine Prozesshaftigkeit (Löw und Sturm 2019), berücksichtigt werden können. Die Analyse der aufgeräumten Kitas und der Fokus auf die von den Kinderbetreuenden organisierten Raumstrukturen verfolgen die Perspektive der Handlungs- und Gestaltungsmacht der Fachpersonen und damit neben den Raumpraktiken in erster Linie den geplanten Raum. Die Raumgestaltung orientiert sich an pädagogischen Konzepten, die häufig den Raum als Akteur mit typischen Umsetzungen nutzen (Knauf 2019). Der als performativ verstandene Raum wurde mittels der Analyse der Erzählungen der Kitaleitungen hinsichtlich der intendierten Verwendung der räumlichen Arrangements einer Analyse zugänglich gemacht, womit auch Raumpraktiken in den Blick genommen werden konnten.

Die Raumanalyse macht die im Raum tradierten Genderismen sichtbar, entsprechend zeigt sich die hohe Aktualität der theoretischen Konzeptionen von Goffman (1977/1994; s. Abschn. 2.1.2). Deutlich wird, dass die Zuteilung von Spielangeboten als Jungen- oder Mädchenspiel die räumliche Anordnung in mehreren Bereichen stärker strukturiert als die ebenfalls traditionellen Zuordnungen zu Spielformen (beispielsweise kleines Rollenspiel) oder Bildungsbereichen (beispielsweise bildnerisches Gestalten). Eine große Zahl an Fachpublikationen für die Praxis fokussiert auf die Raumeinrichtung und die Materialisierung der Spielangebote - das Bereitstellen einer anregenden Lernumgebung wird als eine Kernaufgabe der Kinderbetreuenden zur frühen Bildung betrachtet. Dass die Einteilung nach Genderismen dennoch kaum in den von uns geführten Interviews zur Raumbegehung auffällt, reflektiert und infrage gestellt wird, zeigt anschaulich, was Goffman (1994) beschrieben hat: Genderismen sind institutionell verankert, sie gehören einfach dazu. Sie werden nicht hinterfragt und auch nicht bewusst eingesetzt, sie sind die „Infrastrukturen“ (Hirschauer 2012). Die binäre Geschlechterordnung und die Annahme, dass Jungen und Mädchen unterschiedlich spielen, wird dabei implizit vorausgesetzt. Obwohl in den von uns geführten Interviews mit den Kitaleitungen eine geschlechtsstereotype Beschreibung des kindlichen Spiels kritisch hinterfragt und stereotype Zuordnungen als überkommen identifiziert werden, sind die Genderismen als Differenzvorstellungen nach wie vor im räumlichen Arrangement der Kitas verankert und blieben hier häufig unhinterfragt. 
Es ist anzunehmen, dass diese räumlichen Arrangements Aspekte des doing gender in der Kita fortsetzen und verankern. Auch wenn Kindern und Fachpersonen situativ undoing gender erlaubt wird und dies in den Interaktionen auch auf vielfältige Weise beobachtet wurde (vgl. Kap. 4), vermittelt dennoch die Raumgestaltung und die Anordnung des Spielmaterials in Funktionsecken und -räumen die traditionelle Aufteilung in Mädchen- und Jungenspiel sowie in häuslich-privat und beruflich-öffentlich und setzt diese unhinterfragt als gegeben voraus. Diese Erkenntnisse des Forschungsprojekts erwiesen sich hier auch für die Praxis als sehr bedeutsam, sowohl als ein für Praktikerinnen und Praktiker zugänglicher Einstieg in das Verständnis von doing und undoing gender in der Kita als auch hinsichtlich des damit verbundenen Reflexionspotenzials. Die kritische Überprüfung der räumlichen Arrangements und die darauf initiierte Umgestaltungen der räumlichen Ordnungen von Funktionsecken und Spielmaterial erwies sich hierbei als sehr produktiv.

In den Transferworkshops und im Umsetzungsprojekt (Vogt und Nentwich 2015) erlaubte die Perspektive des (un)doing gender im Raum eine Selbstreflexion der häufig unhinterfragten, tradierten Praxis. In den Workshops wurde angeregt, die Spielmaterialien und Bereiche nach den Spielformen anzuordnen, zum Beispiel kleines Rollenspiel und Bauspiel zusammen, also die Puppenstube zur Garage, und zu den Tieren und neben den Bauernhof.

Weiter wurde eingebracht, die traditionellen Ordnungen der Spielmaterialien und Funktionsecken zu hinterfragen und mehr unstrukturiertes Material wie Häuser, Tücher und Schachteln sowie thematische Anregungen wie beispielsweise ein Baustellenzimmer einzurichten, das Spiel anzuregen und danach wieder einen neuen thematischen Spielbereich einzurichten. Wie Andresen (2011) festhält, ist es für das Spiel der Kinder wichtig, dass realitätsnahe wie auch unbestimmte Objekte zur Verfügung stehen. Solche Requisiten können den Einstieg ins Rollenspiel erleichtern - und zugleich die starre gegenderte Ordnung auflösen.

Die Analyse der räumlichen Arrangements in der Kita auf der Ebene der räumlichen Zuordnung, der Ordnungsprinzipien und der angebotenen Spielmaterialien als Artefakte zeigt, wie sehr Genderismen fortgeschrieben werden. Das Potenzial von undoing gender in der Raumgestaltung wird noch wenig genutzt, obwohl dem Raum in der Pädagogik der frühen Kindheit traditionell eine sehr große Bedeutung zugeschrieben wird.

\section{Literatur}

Acker, Joan (1990). Hierarchies, Jobs, Bodies: A Theory of Gendered Organizations. Gender \& Society, 4(2), 139-158. 
Ämter der Landesregierungen der österreichischen Bundesländer (Hrsg.). (2009). Bundesländerübergreifender BildungsRahmenPlan für elementare Bildungseinrichtungen in Österreich. https://www.charlotte-buehler-institut.at/bundeslaenderuebergreifender-bil dungsrahmenplan-fuer-elementare-bildungseinrichtungen-in-oesterreich-2/.

Andresen, Helga (2011). Erzählen und Rollenspiel von Kindern zwischen drei und sechs Jahren. Eine Expertise der Weiterbildungsinitiative Frühpädagogische Fachkräfte (WiFF). München: Deutsches Jugendinstitut.

Andrey, Stefanie, \& Nyffenegger, Lea (2008). Typisch Mädchen? Typisch Junge? Eine empirische Studie zur Entwicklung geschlechterstereotyper Vorstellungen bei Kindern. Pädagogische Hochschule Bern (Unveröffentlichte studentische Arbeit).

Barrett, Peter, Davies, Fay, Zhang, Yufan, \& Barrett, Lucinda (2017). The Holistic Impact of Classroom Spaces on Learning in Specific Subjects. Environment and Behavior, 49(4), 425-451.

Beebe, Kathryne, Davis, Angela, \& Gleadle, Kathryn (2017). Introduction: Space, Place and Gendered Identities: Feminist History and the Spatial Turn. Women's History Review, 21(4), 523-532.

Berger, Manfred (2000). Frauen in der Geschichte des Kindergartens: Henriette SchraderBreymann. In Martin R. Textor, \& Antje Bostelmann (Hrsg.), Kindergartenpädagogik.de. https://kindergartenpaedagogik.de/fachartikel/geschichte-der-kinderbetreu ung/manfred-berger-frauen-in-der-geschichte-des-kindergartens/159.

BfS. (2017). Männer legen bei Haus- und Familienarbeit zu - Frauen bei bezahlter Arbeit. Medienmitteilung. Neuchâtel: Bundesamt für Statistik. https://www.bfs.admin.ch/bfs/ de/home/statistiken/arbeit-erwerb/unbezahlte-arbeit/haus-familienarbeit.assetdetail.296 7878.html.

Blommaert, Jan (2013). Ethnography, superdiversity and linguistic landscapes: Chronicles of complexity. Bristol: Multilingual Matters.

Braun, Mario, \& Steffes, Daniela (2013). Aus der Rolle fallen. Geschlechtsbewusste Pädagogik in der Bildungspraxis katholischer Familienzentren. Kompakt, 2, 14-17.

Breidenstein, Georg, \& Kelle, Helga (1998). Geschlechteralltag in der Schulklasse. Weinheim: Juventa.

Crowther, Ingrid (2007). Im Kindergarten kreativ und effektiv lernen - auf die Umgebung kommt es an. Berlin: Cornelsen.

Dahlinger, Sarah (2009). Der Raum als dritter Pädagoge. PÄD Forum, 28(37), 247-250.

Dannhauer, Heinz (1977). Geschlecht und Persönlichkeit: eine Untersuchung zur psychischen Geschlechtsdifferenzierung in der Ontogenese. Berlin: Deutscher Verlag der Wissenschaften.

Eckes, Thomas (2008). Geschlechterstereotype: Von Rollen, Identitäten und Vorurteilen. In Ruth Becker, \& Beate Kortendiek (Hrsg.), Handbuch Frauen-und Geschlechterforschung (S. 171-182). Wiesbaden: VS Verlag für Sozialwissenschaften.

Erning, Günter (2004). Bildungsförderung in einem klassischen frühpädagogischen Konzept: Die Entwicklung des Kindergartens. In Gabriele Faust, Margarete Götz, Hartmut Hacker, \& Hans-Günther Rossbach (Hrsg.), Anschlussfähige Bildungsprozesse im Elementar- und Primarbereich (S. 27-48). Bad Heilbrunn: Klinkhardt.

Fooken, Ines (2019). Puppe - Seelengefährtin, Spiegel, Alter Ego, Hilfs-Ich und noch vieles mehr. In Spielzeugmuseum Riehen (Hrsg), Puppen. Eine Sammlung von Doris Im Obersteg-Lerch (S. 96-105). Basel: Schwabe. 
Franz, Margrit, \& Vollmert, Margrit (2005). Raumgestaltung in der Kita: in diesen Räumen fühlen sich Kinder wohl. München: Don Bosco.

Frehse, Fraya (2016). Erving Goffmans Soziologie des Raums. sozialraum.de, 1. https://www. sozialraum.de/erving-goffmans-soziologie-des-raums.php.

Fritzsche, Bettina, Idel, Till-Sebastian, \& Rabenstein, Kerstin (2011). Ordnungsbildung in pädagogischen Praktiken. Praxistheoretische Überlegungen zur Konstitution und Beobachtung von Lernkulturen. Zeitschrift für Soziologie der Erziehung und Sozialisation ZSE, 31(1), 28-44.

Goffman, Erving (1994). Das Arrangement der Geschlechter. In Hubert A. Knoblauch (Hrsg.), Interaktion und Geschlecht (S. 105-158). Frankfurt: Campus Verlag.

Grünewald-Huber, Elisabeth (2009). Geschlechterbezogene Selbstkonzepte und Spielvorlieben von 5- bis 8-Jährigen. Beitrag präsentiert an der SGL-Tagung „Entwicklung und Lernen junger Kinder“. St. Gallen, Schweiz. https://docplayer.org/31490911-Geschlech terbezogene-selbstkonzepte-und-spielvorlieben-von-5-bis-8-jaehrigen.html.

Gulson, Kalervo N., \& Symes, Colin (2007). Knowing one's Place: Space, Theory, Education. Critical Studies in Education, 48(1), 97-110.

Gutknecht, Dorothee (2016). Die Kindergruppe als Ort der Raumerfahrung und Raumaneignung. In Rita Braches-Chyrek, \& Charlotte Röhner (Hrsg.), Kindheit und Raum (S. 145-162). Opladen: Barbara Budrich.

Hauser, Bernhard (2005). Das Spiel als Lernmodus: Unter Druck von Verschulung-im Lichte der neueren Forschung. In Titus Guldimann, \& Bernhard Hauser (Hrsg.), Bildung 4 bis 8 jähriger Kinder (S. 143-167). Münster: Waxmann.

Hauser, Bernhard (2016). Spielen: Frühes Lernen in Familie, Krippe und Kindergarten. Stuttgart: Kohlhammer.

Hinz, Renate (2012). Lernen und leben im Raum. Die Grundschulzeitschrift, 26(255/256), $50-54$.

Hirschauer, Stefan (2006). Putting Things into Words. Ethnographic Description and the Silence of the Social. Human Studies, 29(4), 413-441.

Hirschauer, Stefan (2012). Die Praxis der Geschlechter(in)differenz und ihre Infrastruktur. In Julia Graf, Kristin Ideler, \& Sabine Klinger (Hrsg.), Geschlecht zwischen Struktur und Subjekt: Theorie, Praxis, Perspektiven (S. 153-171). Opladen: Barbara Budrich.

Hoffmann, Cornelia (2013). Emmi Pikler: 'Berührende' Erziehung. In Cornelia Hoffmann, \& Michael Obermaier (Hrsg.), Projekt Frühkindliche Erziehung (S. 161-170). Paderborn: Ferdinand Schöningh.

Imfeld, Claudia (2012) Ich Barbie, du Ken. Beobachter, 6, 66. https://www.beobachter.ch/ familie/kinder/geschlechtertrennung-ich-barbie-du-ken.

Jäger, Marianna (2008). Alltagskultur im Kindergarten. Lebensweltliche Ethnographie aus ethnologischer Perspektive. In Bettina Hünersdorf, Burkhard Müller, \& Christoph Maeder (Hrsg.), Ethnographie und Erziehungswissenschaft. Methodologische Reflexionen und empirische Annäherungen (S. 141-150). Weinheim: Juventa.

Jung, Petra (2009). Kindertageseinrichtungen zwischen pädagogischer Ordnung und den Ordnungen der Kinder. Wiesbaden: VS Verlag für Sozialwissenschaften.

Karsten, Lia (2003). Children's use of public space: the gendered world of the playground. Childhood Education, 10(4), 457-473. 
Kasüschke, Dagmar (2016). Die Kindertageseinrichtung als Raum pädagogisch inszenierter Kindheit. In Rita Braches-Chyrek, \& Charlotte Röhner (Hrsg.), Kindheit und Raum (S. 180-198). Opladen: Barbara Budrich.

Knauf, Helen (2017). Visuelle Raumanalyse. Frühe Bildung, 6(1), 33-40.

Knauf, Helen (2019). Visual Environmental Scale: Analysing the Early Childhood Education Environment Early Childhood Education Journal, 47(1), 43-51.

Kogel, Katrin (2007). Kindern Raum für Erfahrungen geben. Klein \& gross, 12, 12-16.

Kubandt, Melanie (2016). Geschlechterdifferenzierung in der Kindertageseinrichtung: Eine qualitativ-rekonstruktive Studie. Opladen: Barbara Budrich.

Kuckartz, Uwe (2012). Qualitative Inhaltsanalyse. Weinheim: Beltz.

Kuhn, Melanie (2013). Professionalität im Kindergarten. Eine ethnographische Studie zur Elementarpädagogik in der Migrationsgesellschaft. Wiesbaden: VS Verlag für Sozialwissenschaften.

Lefèbvre, Henri (1991). The Production of Space. Cambridge: Blackwell.

Löw, Martina (2008). The Constitution of Space. The Structuration of Spaces through the Simultaneity of Effect and Perception. European Journal of Social Theory, 11(1), 25-49.

Löw, Martin, \& Sturm, Gabriele (2019). Raumsoziologie. In Fabian Kessl, \& Christian Reutlinger (Hrsg.), Handbuch Sozialraum (S. 3-22). Wiesbaden: VS Verlag für Sozialwissenschaften.

Mahlke, Wolfgang (2003). Mehr Lebensqualität durch sorgsame Raumgestaltung (1). Spektrum, 44-47.

Moreno-Colom, Sara (2017). The Gendered Division of Housework Time: Analysis of Time Use by Type and Daily Frequency of Household Tasks. Time \& Society, 26(1), 3-27.

Nentwich, Julia C., Vogt, Franziska, \& Tennhoff, Wiebke (2016). Care and Education? Exploring the Gendered Rhythms and Routines of Childcare Work. In Brigitte Liebig, Karin Gottschall, \& Birgit Sauer (Hrsg.), Gender Equality in Context: Policies and Practices in Switzerland (S. 217-237). Opladen: Barbara Budrich.

Pink, Sarah (2007). Doing Visual Ethnography. London: Sage.

Pink, Sarah (2008). Mobilising Visual Ethnography: Making Routes, Making Place and Making Images. Forum Qualitative Sozialforschung/Forum: Qualitative Social Research, 9(4). https://www.qualitative-research.net/index.php/fqs/article/view/1166/2581.

Pütz, Tanja., \& Klein-Landeck, Michael (2019). Montessori-Pädagogik: Einführung in Theorie und Praxis. Freiburg: Herder.

Rabe-Kleberg, Ursula (2003). Gender Mainstreaming und Kindergarten. Weinheim: Beltz.

Reyer, Jürgen, \& Franke-Meyer, Diana (2016). Räume und Räumlichkeiten in der Geschichte der Pädagogik der frühen Kindheit und des Kindergartens. In Rita Braches-Chyrek, \& Charlotte Röhner (Hrsg.), Kindheit und Raum (S. 163-179). Opladen: Barbara Budrich.

Rohrmann, Tim (2009). Gender in Kindertageseinrichtungen. Ein Überblick über den Forschungsstand. München: Deutsches Jugendinstitut. https://www.dji.de/fileadmin/user_u pload/bibs/Tim_Rohrmann_Gender_in_Kindertageseinrichtungen.pdf.

Sarbach, Sonja (2011). Norminventar Kindergarten: Lehr-und Lernmittelliste. St.Gallen: Pädagogische Kommission I, Kindergarten, Kanton St. Gallen. https://docplayer.org/113 05041-Norminventar-kindergarten-lehr-und-lernmittelliste.html.

Stieve, Claus (2013). Differenzen früher Bildung in der Begegnung mit den Dingen: Am Beispiel des Wohnens und seiner Repräsentation im Kindergarten. Zeitschrift für Erziehungswissenschaft, 16(2), 91-106. 
Tennhoff, Wiebke, Nentwich, Julia C., \& Vogt, Franziska (2014). Gender in der Kita. Praxisratgeber für Kitaleitungen. St. Gallen: Universität und Pädagogische Hochschule St. Gallen. www.gender-kita.ch.

Thorne, Barrie (1992/2007). Girls and Boys Together... But Mostly Apart: Gender Arrangements in Elementary Schools. In James M. Henslin (Ed.), Life in society (pp. 115-130). Boston: Pearson (Erstveröffentlichung 1992).

Van Dieken, Christel, \& Rohrmann, Tim (2003). Raum und Räume für Mädchen und Jungen. Angebote und Raumnutzung unter geschlechtsspezifischen Aspekten. Kindergarten Heute, 33(1), 26-33.

Vogt, Franziska (2002). No Ethnography Without Comparison: The Methodological Significance of Comparison in Ethnographic Research. In Geoffrey Walford (Ed.), Debates and developments in ethnographic methodology (pp. 23-42). Oxford: JAI und Elsevier Science.

Vogt, Franziska, Nentwich, Julia C., Poppen, Wiebke, \& Schälin, Stefanie (2013). Offiziersmütze und Stöckelschuhe. Bildung Schweiz, 10, 42-43.

Vogt, Franziska \& Nentwich, Julia C. (2015). Gender in der Kita: Veränderung zur Inklusion von Männern gemeinsam gestalten. Projekt 14-018, unterstützt durch das Eidgenössische Büro für Gleichstellung von Frau und Mann. Projektwebseite: www.gender-kita.ch.

von der Beek, Angelika (2014). Bildungsräume für Kinder von null bis drei. Kiliansroda: Verlag Das Netz.

Walter-Laager, Catherine (Hrsg.) (2015). Erfahrungsfelder und Beobachtungspunkte für den Frühbereich. Zürich: Stadt Zürich. https://www.stadt-zuerich.ch/content/dam/stzh/ssd/ Deutsch/Volksschule/dokumente/publikationen_broschueren/fruehfoerderung/dossier_e rfahrungsfelder.pdf.

Wasserman, Varda, \& Frenkel, Michal (2015). Spatial Work in Between Glass Ceilings and Glass Walls: Gender-Class Intersectionality and Organizational Aesthetics. Organization Studies, 36(11), 1485-1505.

Wilkening, Friedrich (2004). Kindliches Erfassen von Raum und Zeit. 4 bis 8 Fachzeitschrift für Kindergarten und Unterstufe, 12, 14-15.

Wustmann Seiler, Corina, \& Simoni, Heidi (2012). Orientierungsrahmen für frühkindliche Bildung, Betreuung und Erziehung in der Schweiz. Zürich: Marie Meierhofer Institut für das Kind im Auftrag der Schweizerischen UNESCO-Kommission und des Netzwerks Kinderbetreuung Schweiz. https://www.netzwerk-kinderbetreuung.ch/de/publikati onen/20/. 
Open Access Dieses Kapitel wird unter der Creative Commons Namensnennung 4.0 International Lizenz (http://creativecommons.org/licenses/by/4.0/deed.de) veröffentlicht, welche die Nutzung, Vervielfältigung, Bearbeitung, Verbreitung und Wiedergabe in jeglichem Medium und Format erlaubt, sofern Sie den/die ursprünglichen Autor(en) und die Quelle ordnungsgemäß nennen, einen Link zur Creative Commons Lizenz beifügen und angeben, ob Änderungen vorgenommen wurden.

Die in diesem Kapitel enthaltenen Bilder und sonstiges Drittmaterial unterliegen ebenfalls der genannten Creative Commons Lizenz, sofern sich aus der Abbildungslegende nichts anderes ergibt. Sofern das betreffende Material nicht unter der genannten Creative Commons Lizenz steht und die betreffende Handlung nicht nach gesetzlichen Vorschriften erlaubt ist, ist für die oben aufgeführten Weiterverwendungen des Materials die Einwilligung des jeweiligen Rechteinhabers einzuholen.

(c) (9) 